\title{
Team-Optimal Distributed MMSE Estimation in General and Tree Networks
}

\author{
Muhammed O. Sayin ${ }^{\mathrm{a}, *}$, Suleyman S. Kozat ${ }^{\mathrm{b}}$, Tamer Başar ${ }^{\mathrm{a}}$ \\ ${ }^{a}$ The Department of Electrical and Computer Engineering, University of Illinois at \\ Urbana-Champaign, Champaign, IL 61801 USA \\ ${ }^{b}$ The Department of Electrical and Electronics Engineering, Bilkent University, Bilkent, \\ Ankara 06800 Turkey
}

\begin{abstract}
We construct team-optimal estimation algorithms over distributed networks for state estimation in the finite-horizon mean-square error (MSE) sense. Here, we have a distributed collection of agents with processing and cooperation capabilities. These agents observe noisy samples of a desired state through a linear model and seek to learn this state by interacting with each other. Although this problem has attracted significant attention and been studied extensively in fields including machine learning and signal processing, all the well-known strategies do not achieve team-optimal learning performance in the finite-horizon MSE sense. To this end, we formulate the finite-horizon distributed minimum MSE (MMSE) when there is no restriction on the size of the disclosed information, i.e., oracle performance, over an arbitrary network topology. Subsequently, we show that exchange of local estimates is sufficient to achieve the oracle performance only over certain network topologies. By inspecting these network structures, we propose recursive algorithms achieving the oracle performance through the disclosure of local estimates. For practical implementations we also provide approaches to reduce the complexity of the algorithms through the time-windowing of the observations. Finally, in the numerical examples, we demonstrate the superior performance of the introduced algorithms in the
\end{abstract}

\footnotetext{
${ }^{*}$ Corresponding author

Email addresses: sayin2@illinois.edu (Muhammed O. Sayin),

kozat@ee.bilkent.edu.tr (Suleyman S. Kozat), basar1@illinois.edu (Tamer Başar)
}

Preprint submitted to Journal of ${ }^{A} T_{E} X$ Templates

January 8, 2017

(C) 2017. This manuscript version is made available under the Elsevier user license http://www.elsevier.com/open-access/userlicense/1.0/ 
finite-horizon MSE sense due to optimal estimation.

Keywords: Distributed networks, distributed Kalman filter, optimal information disclosure, team problem, finite-horizon, MMSE estimation, tree networks, Gaussian processes.

\section{Introduction}

Over a distributed network of agents with measurement, processing and communication capabilities, we can have enhanced processing performance, e.g., fast response time, relative to the centralized networks by distributing the process5 ing power over the networks [1, 2, 3, 4. Mainly, distributed agents observe the true state of the system through noisy measurements from different viewpoints, process the observation data in order to estimate the state, and communicate with each other to alleviate the estimation process in a fully distributed manner. Notably, the agents can respond to streaming data in an online manner by disclosing information among each other at certain instances. This framework is conveniently used to model highly complex structures from defense applications to social and economical networks [5, 6, 7, 8. As an example, say that we have radar systems distributed over an area and seeking to locate hostile missiles, i.e., the location of the missile is the underlying state of the system. In that respect, distributed processing approach has vital importance in terms of detecting the missiles and reacting as fast as possible. In particular, even if the viewpoints of a few radar systems are blocked due to environmental obstacles, through the communication among the radar systems, each system should still be able to locate the missiles. Additionally, since each radar system not only collects measurements but also process them to locate the missiles, the overall system can respond to the missiles faster than a centralized approach in which measurements of all the radar systems are collected at a centralized unit and processed together.

Although there is an extensive literature on this topic, e.g., [2, 6, 7, 8, 9, 25 10, 11 and references therein, we still have significant and yet unexplored prob- 
lems for disclosure and utilization of information among agents. Prior work has focused on the computationally simple algorithms that aim to minimize certain cost functions through the exchange of local estimates, e.g., diffusion or consensus based estimation algorithms [2, 9, 3, 12, 13, 14, due to processso ing power related practical concerns. However, there is a trade-off in terms of computational complexity and estimation performance.

Formulating the optimal distributed estimation algorithms with respect to certain performance criteria is a significant and unexplored challenge. To this end, we consider here the distributed estimation problem as a team problem for distributed agents in which agents take actions, e.g., which information to disclose and how to construct the local estimate. This differs from the existing approaches in which agents exchange their local estimates. Furthermore, we address the optimality of exchanging local estimates with respect to the team problem over arbitrary network structures.

40 We examine the optimal usage of the exchanged information based on its content rather than a blind approach in which exchanged information is handled irrespective of the content as in the diffusion or consensus based approaches. In such approaches, the agents utilize the exchanged information generally through certain static combination rules, e.g., the uniform rule [15], the Laplacian rule 45 [16] or the Metropolis rule [17. However, if the statistical profile of the measurement data varies over the network, i.e., each agent observes diverse signalto-noise ratios, by ignoring the variation in noise, these rules yield severe degradation in the estimation performance [2]. In such cases the agents can perform better even without cooperation 22. Therefore, the optimal usage of the exchanged information plays an essential role in performance improvement in the team problem.

Consider distributed networks of agents that observe noisy samples of an underlying state (possibly multi-dimensional) over a finite horizon. The agents can exchange information with only certain other agents at each time instant.

${ }_{55}$ In particular, agents cooperate with each other as a team according to a certain team cost depending on the agents' actions. To this end, each agent constructs a 
local estimate of the underlying state and constructs messages to disclose to the neighboring agents at each time instant. We particularly consider a quadratic cost function and that the underlying state and measurement noises are jointly 60 Gaussian.

We note that restrictions on the sent messages, e.g., on the size of the disclosed information, has significant impact on the optimal team actions. We introduce the concept of the oracle performance, in which there is no restriction on the disclosed information. In that case, a time-stamped information disclosure can be team-optimal and we introduce the optimal distributed online learning (ODOL) algorithm using the time-stamped information disclosure. Through a counter example, we show that the oracle performance cannot be achieved through the exchange of local estimates. Then, we analytically show that over certain networks, e.g., tree networks, agents can achieve the oracle performance through the exchange of local estimates. We propose the optimal and efficient distributed online learning (OEDOL) algorithm, which is practical for real life applications and achieves the oracle performance over tree networks through the exchange of local estimates. Finally, we introduce the time windowing of the measurements in the team cost and propose a recursive algorithm, sub-optimal distributed online learning (SDOL) algorithm, combining the received messages linearly through time-invariant combination weights.

We can list our main contributions as follows: 1) We introduce a teamproblem to minimize finite horizon mean square error cost function in a distributed manner. 2) We derive the ODOL algorithm achieving the oracle performance over arbitrary networks through time-stamped information exchange. 3) We address whether agents can achieve the oracle performance through the disclosure of local estimates. 4) We propose a recursive algorithm, the OEDOL algorithm, achieving the oracle performance over certain network topologies with tremendously reduced communication load. 5) We also formulate suboptimal versions of the algorithms with reduced complexity. 6) We provide numerical examples demonstrating the significant gains due to the introduced algorithms. 


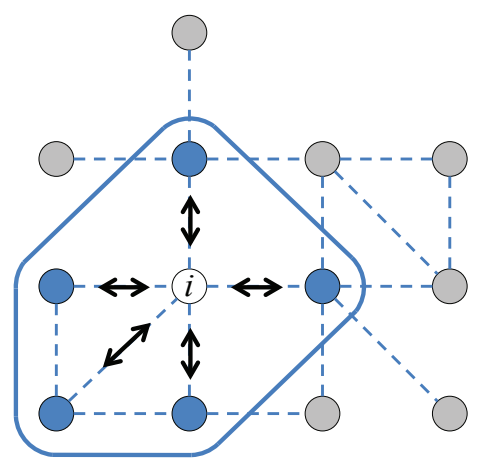

(a) First-order neighborhood

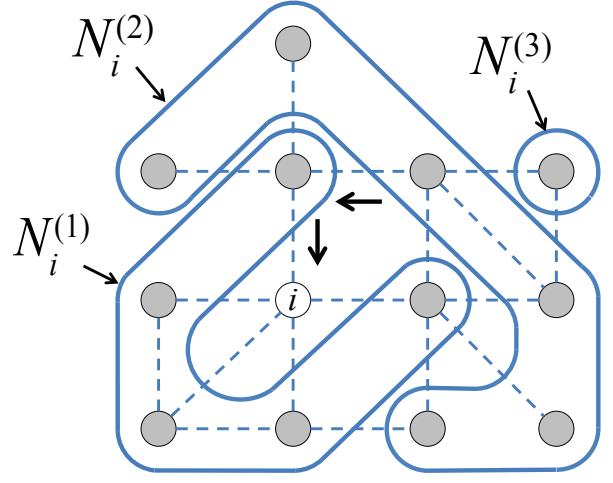

(b) All neighborhoods

Figure 1: The neighborhoods of $i$ th agent over the distributed network.

The remainder of the paper is organized as follows. We introduce the team problem for distributed-MMSE estimation in Section 2. We study the tree networks, exploit the network topology to formulate the OEDOL algorithm that reduces the communication load and introduce cell structures, which is relatively more connected than tree networks, in Section 3. We propose the sub-optimal versions of the ODOL algorithm for practical implementations in Section 4. In Section 5, we provide numerical examples demonstrating significant gains due to the introduced algorithms. We conclude the paper in Section 6 with several remarks.

Notation: We work with real data for notational simplicity. $\mathbb{N}(0,$.$) denotes$ the multivariate Gaussian distribution with zero mean and designated covariance. For a vector $a$ (or matrix $A$ ), $a^{\prime}$ (or $A^{\prime}$ ) is its ordinary transpose. We denote the vector whose terms are all 1s (or all 0s) by $\mathbf{1}$ (and $\mathbf{0}$ ). We denote random variables by bold lower case letters, e.g., $\boldsymbol{x}$. The operator $\operatorname{col}\{\cdot\}$ produces a column vector or a matrix in which the arguments of $\operatorname{col}\{\cdot\}$ are stacked one under the other. For a matrix $A, \operatorname{diag}\{A\}$ operator constructs a diagonal matrix with the diagonal entries of $A$. For a given $\operatorname{set} \mathcal{N}, \operatorname{diag}\{\mathcal{N}\}$ creates a diagonal matrix whose diagonal block entries are elements of the set. The operator $\otimes$ denotes the Kronecker product. 


\section{Team Problem for Distributed-MMSE Estimation}

Consider a distributed network of $m$ agents with processing and communication capabilities. In Fig. 1, we illustrate this network through an undirected agents as seen in Fig. 1a. Let $z_{i, j, t} \in \mathbb{R}^{r}$ denote the information disclosed by $i$ to $j$ at time $t$, and $r \geq 0$ is the size of the disclosed information. We assume

\footnotetext{
${ }^{1}$ For notational simplicity, we define $\mathcal{N}_{i}:=\mathcal{N}_{i}^{(1)}$ and $\pi_{i}:=\pi_{i}^{(1)}$
} 
that there exists a perfect channel between the agents such that the disclosed information can be transmitted with infinite precision. Therefore, we denote the information available to agent- $i$ at time $t$ by

$$
\delta_{i, t}=\left\{y_{i, t}, y_{i, \tau}, z_{j, i, \tau}, \text { for } j \in \mathcal{N}_{i}, \tau=1, \cdots, t-1\right\}
$$

and let $\sigma_{i, t}$ denote the sigma-algebra generated by the information set $\delta_{i, t}$. Furthermore, we define the set of all $\sigma_{i, t}$-measurable functions from $\mathbb{R}^{q t} \times \mathbb{R}^{r \pi_{i}(t-1)}$ to $\mathbb{R}^{r}$ by $\Gamma_{i, t}$. Importantly, here, which information to disclose is not determined a priori in the problem formulation. Let $\gamma_{i, j, t}$ be the decision strategy for $z_{i, j, t}$, then agent- $i$ chooses $\gamma_{i, j, t}, j \in \mathcal{N}_{i}$, from the set $\Gamma_{i, t}$, i.e., $\gamma_{i, j, t} \in \Gamma_{i, t}$ and $\gamma_{i, j, t}\left(\delta_{i, t}\right)=z_{i, j, t}$, based on his/her objective.

In addition to the disclosed information $z_{i, j, t}, j \in \mathcal{N}_{i}$, agent- $i$ takes action $u_{i, t} \in \mathbb{R}^{q}$, where the corresponding decision strategy $\eta_{i, t}$ is chosen from the set $\Omega_{i, t}$, which is the set of all $\sigma_{i, t}$-measurable functions from $\mathbb{R}^{q t} \times \mathbb{R}^{r \pi_{i}(t-1)}$ to $\mathbb{R}^{p}$, ${ }_{145}$ i.e., $\eta_{i, t} \in \Omega_{i, t}$ and $\eta_{i, t}\left(\delta_{i, t}\right)=u_{i, t}$. Here, we consider that the agents have a common cost function:

$$
\sum_{t=1}^{T} \sum_{j=1}^{m}\left\|x-u_{j, t}\right\|^{2}
$$

where all actions $u_{i, t}, i=1, \cdots, m$ and $t=1, \cdots, T$ are costly, and agent- $i$ should take actions $u_{i, t}$ and $z_{i, j, t}, j=1, \cdots, \pi_{i}$ and $t=1, \cdots, T$, accordingly. Therefore, this corresponds to a team-problem, in which agent- $i$ faces the following minimization problem:

$$
\min _{\substack{\gamma_{i, j, t} \in \Gamma_{i, t}, \eta_{i, t} \in \Omega_{i, t}, j \in \mathcal{N}_{i}, t=1, \cdots, T}} \sum_{t=1}^{T} \sum_{j=1}^{m} E\left\|\boldsymbol{x}-\eta_{j, t}\left(\delta_{j, t}\right)\right\|^{2} .
$$

We point out that both $\Gamma_{i, t}$ and $\Omega_{i, t}$ are infinite dimensional, i.e., (1) is a functional optimization problem and the optimal strategies can be a nonlinear function of the available information. Furthermore, the agents should also construct the disclosed information accordingly since other agents' decisions $u_{j, t}$ directly depend on the disclosed information. 


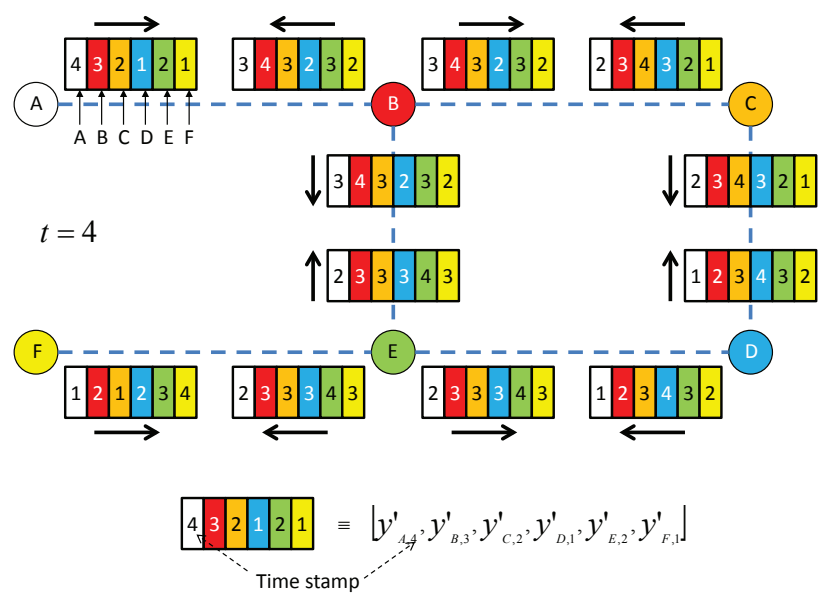

Figure 2: Time stamped information disclosure over a network of 6 agents at time instant $t=4$.

\subsection{A Lower Bound}

In order to compute the team optimal strategies, we first construct a lower bound on the performance of the agents by removing the limitation on the size of the disclosed information, i.e., $r \rightarrow \infty$. In that case, the following proposition provides an optimal information disclosure strategy.

Proposition 2.1. When $r \rightarrow \infty$, a time stamped information disclosure strategy, in which agents transmit the most current version of the available information (e.g., see Fig. 2), can lead to the team-optimal solution.

Proof. Through the time stamped information disclosure, each agent can obtain the measurements of the other agents separately in a connected network. However, the measurements of the non-neighboring agents could only be received after certain hops due to the partially connected structure, i.e., certain agents are not directly connected. As an example, the disclosed information of $j \in \mathcal{N}_{i}^{(2)}$ reaches to $i$ by passing through two communication links as seen in Fig. $1 \mathrm{~b}$ In particular, this case assumes that each agent has access to full information from the other agents, albeit with certain hops, and corresponds to the direct aggregation of all measurements across the network at each agent. 
Correspondingly, at time $t$, all the information aggregated at $i$ th agent is given by

$$
\delta_{i, t}^{o}:=\left\{\left\{y_{i, \tau}\right\}^{\tau \leq t},\left\{y_{j, \tau}\right\}_{j \in \mathcal{N}_{i}^{(1)}}^{\tau \leq t-1}, \cdots,\left\{y_{j, \tau}\right\}_{\substack{\tau \leq t-\kappa_{i} \\ j \in \mathcal{N}_{i}^{\left(\kappa_{i}\right)}}}^{\left(\kappa^{\prime}\right.}\right\},
$$

following minimization problem:

$$
\min _{\substack{\eta_{i, t} \in \Omega_{i, t}^{o}, t=1, \cdots, T}} \sum_{t=1}^{T} \sum_{j=1}^{m} E\left\|\boldsymbol{x}-\eta_{j, t}\left(\delta_{j, t}^{o}\right)\right\|^{2},
$$

which is equivalent to

$$
\sum_{t=1}^{T} \min _{\eta_{i, t} \in \Omega_{i, t}^{o}} E\left\|\boldsymbol{x}-\eta_{i, t}\left(\delta_{i, t}^{o}\right)\right\|^{2}
$$

since $\gamma_{i, j, t}, j \in \mathcal{N}_{i}$, is set to the time-stamped strategy and $\eta_{i, t}$ has impact only on the term $E\left\|\boldsymbol{x}-\eta_{i, t}\left(\delta_{i, t}^{o}\right)\right\|^{2}$. Let $\boldsymbol{\delta}_{i, t}^{o}$ be defined by

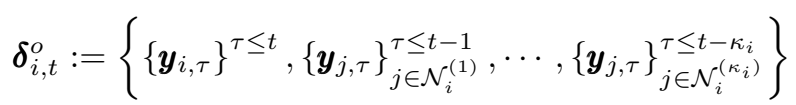

Then, team optimal strategy in the lower bound, i.e., oracle strategy, $\eta_{i, t}^{o}$ and the corresponding action $u_{i, t}^{o}$ are given by

$$
\eta_{i, t}^{o}\left(\delta_{i, t}^{o}\right)=u_{i, t}^{o}=E\left[\boldsymbol{x} \mid \boldsymbol{\delta}_{i, t}^{o}=\delta_{i, t}^{o}\right]
$$

and we define $\boldsymbol{u}_{i, t}^{o}:=E\left[\boldsymbol{x} \mid \boldsymbol{\delta}_{i, t}^{o}\right]$.

\section{2. $O D O L$ Algorithm}

Since the state and the observation noise are jointly Gaussian random parameters, we can compute (5) through a Kalman-like recursion [19]. Therefore, we provide the following ODOL algorithm. We introduce a difference set 
$\Delta_{i, t}:=\delta_{i, t}^{o} \backslash \delta_{i, t-1}^{o}=\left\{y_{i, t},\left\{y_{j, t-1}\right\}_{j \in \mathcal{N}_{i}^{(1)}}, \cdots,\left\{y_{j, t-\kappa_{i}}\right\}_{j \in \mathcal{N}_{i}^{\left(\kappa_{i}\right)}}\right\}$ and a vector $w_{i, t}=\operatorname{col}\left\{\Delta_{i, t}\right\}$. Then, for $t \geq 1$ the iterations of the ODOL algorithm are given by

$$
\begin{aligned}
u_{i, t}^{o} & =\left(I-K_{i, t} \bar{H}_{i}\right) u_{i, t-1}^{o}+K_{i, t} w_{i, t}, \\
K_{i, t} & =\hat{\Sigma}_{i, t-1} \bar{H}_{i}^{T}\left(\bar{H}_{i} \hat{\Sigma}_{i, t-1} \bar{H}_{i}^{T}+\bar{\Sigma}_{n_{i}}\right)^{-1}, \\
\hat{\Sigma}_{i, t} & =\left(I-K_{i, t} \bar{H}_{i}\right) \hat{\Sigma}_{i, t-1},
\end{aligned}
$$

where $^{2} u_{i, 0}^{o}=\bar{x}, \hat{\Sigma}_{i, 0}=\Sigma_{x}, \bar{H}_{i}:=\left(P_{i} \otimes I_{q}\right) H, H:=\operatorname{col}\left\{H_{1}, \cdots, H_{m}\right\}$, $\bar{\Sigma}_{n_{i}}:=\left(P_{i} \otimes I_{p}\right) \Sigma_{n}\left(P_{i} \otimes I_{p}\right)^{T}, \Sigma_{n}:=\operatorname{diag}\left\{\Sigma_{n_{1}}, \cdots, \Sigma_{n_{m}}\right\}$, and $P_{i}$ is the corresponding permutation matrix.

We point out that this is a lower bound on the original cost function (1), i.e.,

$$
\sum_{t=1}^{T} \sum_{i=1}^{m} E\left\|\boldsymbol{x}-u_{i, t}^{o}\right\|^{2} \leq \min _{\substack{\gamma_{i, j, t} \in \Gamma_{i, t}, \eta_{i, t} \in \Omega_{i, t}, i=1, \cdots, m ; j \in \mathcal{N}_{i} ; t=1, \cdots, T}} \sum_{t=1}^{T} \sum_{j=1}^{m} E\left\|\boldsymbol{x}-\eta_{j, t}\left(\delta_{j, t}\right)\right\|^{2},
$$

where we substitute team-optimal action (when $r \rightarrow \infty$ ) $u_{i, t}^{o}$ back into (4) and sum over $t=1, \cdots, T$ and $i=1, \cdots, m$. However, the lower bound is not necessarily tight depending on $r$. By Proposition 2.1, time-stamped information disclosure strategy, in which the size of the disclosed information is $q \times m$, yields the oracle solution. This implies that when $r \geq q m$, the lower bound is tight. Furthermore, team optimal solutions are linear in the available information and can be constructed through the recursive algorithm ODOL. However, $q m$ is linear in the number of agents, $m$, and in large networks this can cause excessive communication load yet communication load is crucial for the applicability of the distributed learning algorithms [14, 13. Therefore, in the following section, we provide a sufficient condition on the size of the disclosed information, which depends on the network structure (rather than its size), in order to achieve the lower bound 6 .

\footnotetext{
${ }^{2}$ If the inverse fails to exist, a pseudo inverse can replace the inverse [19].
} 


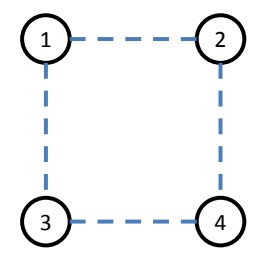

Figure 3: A cycle network of 4 agents.

\section{Distributed-MMSE Estimation with Disclosure of Local Estimate}

In the conventional distributed estimation algorithms, e.g., consensus and diffusion approaches, agents disclose their local estimates, which have size $p$ (note that this does not depend on the network size). The following example addresses whether the disclosure of local estimates can achieve the lower bound (6) or not.

\subsection{A Counter Example}

Consider a cycle network of 4 agents as seen in Fig. 3. where $p=q=1$, $H_{i}=1, \Sigma_{n_{i}}=\sigma_{n}^{2}$, for $i=1, \cdots, 4$, and $\Sigma_{x}=\sigma_{x}^{2}$. We aim to show that agent1 's oracle action at time $t=3$, i.e., $u_{1,3}^{o}$, cannot be constructed through the exchange of local estimates.

At time $t=2$, agent- 2 and agent-3 have the following oracle actions:

$$
\begin{aligned}
u_{2,2}^{o} & =E\left[\boldsymbol{x} \mid \boldsymbol{y}_{2,2}=y_{2,2}, \boldsymbol{y}_{2,1}=y_{2,1}, \boldsymbol{y}_{1,1}=y_{1,1}, \boldsymbol{y}_{4,1}=y_{4,1}\right], \\
& =\frac{\sigma_{x}^{2}}{4\left(\sigma_{x}^{2}+\sigma_{n}^{2}\right)}\left(y_{2,2}+y_{2,1}+y_{1,1}+y_{4,1}\right), \\
u_{3,2}^{o} & =E\left[\boldsymbol{x} \mid \boldsymbol{y}_{3,2}=y_{3,2}, \boldsymbol{y}_{3,1}=y_{3,1}, \boldsymbol{y}_{1,1}=y_{1,1}, \boldsymbol{y}_{4,1}=y_{4,1}\right] . \\
& =\frac{\sigma_{x}^{2}}{4\left(\sigma_{x}^{2}+\sigma_{n}^{2}\right)}\left(y_{3,2}+y_{3,1}+y_{1,1}+y_{4,1}\right) .
\end{aligned}
$$

Note that since there are two hops between agents 2 and 3 , at $t=2$, agents do not have access to each other's any measurement yet. At time $t=3$, agent-1's 
oracle action is given by

$$
\begin{gathered}
u_{1,3}^{o}=E\left[\boldsymbol{x} \mid \boldsymbol{y}_{1,3}=y_{1,3}, \boldsymbol{y}_{1,2}=y_{1,2}, \boldsymbol{y}_{1,1}=y_{1,1},\right. \\
\boldsymbol{y}_{2,2}=y_{2,2}, \boldsymbol{y}_{2,1}=y_{2,1}, \\
\boldsymbol{y}_{3,2}=y_{3,2}, \boldsymbol{y}_{3,1}=y_{3,1}, \\
\left.\boldsymbol{y}_{4,1}=y_{4,1}\right] \\
=\frac{\sigma_{x}^{2}}{8\left(\sigma_{x}^{2}+\sigma_{n}^{2}\right)}\left(y_{1,3}+y_{1,2}+y_{1,1}+y_{2,2}+y_{2,1}+y_{3,2}+y_{3,1}+y_{4,1}\right) .
\end{gathered}
$$

Assume that $u_{1,3}^{o}$ can be obtained through the exchange of local estimates:

$$
\begin{aligned}
\hat{u}_{1,3}:=E\left[\boldsymbol{x} \mid \boldsymbol{y}_{1,3}\right. & =y_{1,3}, \boldsymbol{y}_{1,2}=y_{1,2}, \boldsymbol{y}_{1,1}=y_{1,1}, \\
\boldsymbol{u}_{2,2}^{o} & =u_{2,2}^{o}, \boldsymbol{u}_{2,1}^{o}=u_{2,1}^{o}, \\
\boldsymbol{u}_{3,2}^{o} & \left.=u_{3,2}^{o}, \boldsymbol{u}_{3,1}^{o}=u_{3,1}^{o}\right] .
\end{aligned}
$$

Since all parameters are jointly Gaussian, the local estimates are also jointly Gaussian, $\hat{u}_{1,3}$, is linear in $u_{2,2}^{o}$ and $u_{3,2}^{o}$. Furthermore, the measurements $y_{2,2}$, $y_{3,2}$, and $y_{4,1}$ are only included in $u_{2,2}^{o}$ and $u_{3,2}^{o}$. Therefore, we obtain

$$
\begin{aligned}
\hat{u}_{1,3} & =\cdots+\alpha u_{2,2}^{o}+\beta u_{3,2}^{o} \\
& =\cdots+\alpha \frac{\sigma_{x}^{2}}{4\left(\sigma_{x}^{2}+\sigma_{n}^{2}\right)}\left(y_{2,2}+y_{4,1}+\cdots\right)+\beta \frac{\sigma_{x}^{2}}{4\left(\sigma_{x}^{2}+\sigma_{n}^{2}\right)}\left(y_{3,2}+y_{4,1}+\cdots\right),
\end{aligned}
$$

where $\cdots$ refers to the other terms. However, the equality of $\hat{u}_{1,3}$ and $u_{1,3}^{o}$ implies $\alpha=\beta=1 / 2$ due to the combination weights of $y_{2,2}$ and $y_{3,2}$, respectively, and $\alpha+\beta=1 / 2$ due to the combination weight of $y_{4,1}$, which leads to a contradiction. Hence, which information to disclose over arbitrary networks for team-optimal solutions should be considered elaborately. In the following, we analytically show that lower bound could be achieved through the disclosure of local estimates over "tree networks".

\subsection{Tree Networks}

A network has a "tree structure" if its corresponding graph is a tree, i.e., con- 
star or line networks have tree structures. We remark that for an arbitrary network topology we can also construct the spanning tree of the network and eliminate the cycles. In the literature, there exists numerous distributed algorithms for minimum spanning tree construction [21, 22, 23, 24, 25]. achieve the performance of the oracle algorithm through the disclosure of the local estimates only.

Theorem 3.1: Consider the team-problem over a tree network, in which $r=p$. Then, exchange of local estimates can lead to the team-optimal solution, i.e., agents can achieve the oracle performance.

Proof: Initially, agent- $i$ has access to $y_{i, 1}$ only and the oracle action is $u_{i, 1}^{o}=$ $E\left[\boldsymbol{x} \mid \boldsymbol{y}_{i, 1}=y_{i, 1}\right]$. At time $t=2$, the oracle action is given by

$$
u_{i, 2}^{o}=E\left[\boldsymbol{x} \mid\left\{\boldsymbol{y}_{i, \tau}=y_{i, \tau}\right\}_{\tau=1,2},\left\{\boldsymbol{y}_{j, 1}=y_{j, 1}\right\}_{j \in \mathcal{N}_{i}}\right],
$$

which can be written as

$$
\begin{aligned}
u_{i, 2}^{o} & =E\left[\boldsymbol{x} \mid\left\{\boldsymbol{y}_{i, \tau}=y_{i, \tau}\right\}_{\tau=1,2},\left\{E\left[\boldsymbol{x} \mid \boldsymbol{y}_{j, 1}=y_{j, 1}\right]\right\}_{j \in \mathcal{N}_{i}}\right], \\
& =E\left[\boldsymbol{x} \mid\left\{\boldsymbol{y}_{i, \tau}=y_{i, \tau}\right\}_{\tau=1,2},\left\{\boldsymbol{u}_{j, 1}^{o}=u_{j, 1}^{o}\right\}_{j \in \mathcal{N}_{i}}\right] .
\end{aligned}
$$

This implies that for $t=1$ and $t=2$, the oracle performance can be achieved through the disclosure of local estimate. Therefore, we can consider the oracle action 10 even though agents disclose their local estimate instead of timestamped information disclosure.

As seen in Fig. 4, over a tree network, for $k \in\left\{1, \cdots, \kappa_{i}\right\}$ we have

$$
\mathcal{N}_{i}^{(k)}=\bigcup_{j \in \mathcal{N}_{i}}\left(\mathcal{N}_{i}^{(k)} \cap \mathcal{N}_{j}^{(k-1)}\right) .
$$

Note that the sets in 12 are disjoint as

$$
\left(\mathcal{N}_{i}^{(k)} \cap \mathcal{N}_{j_{1}}^{(k-1)}\right) \cap\left(\mathcal{N}_{i}^{(k)} \cap \mathcal{N}_{j_{2}}^{(k-1)}\right)=\varnothing
$$

for all $j_{1}, j_{2} \in \mathcal{N}_{i}$ and $j_{1} \neq j_{2}$. Notably, over a tree network, by (13), we can partition the collection set of the measurements received after at least $k$-hops 


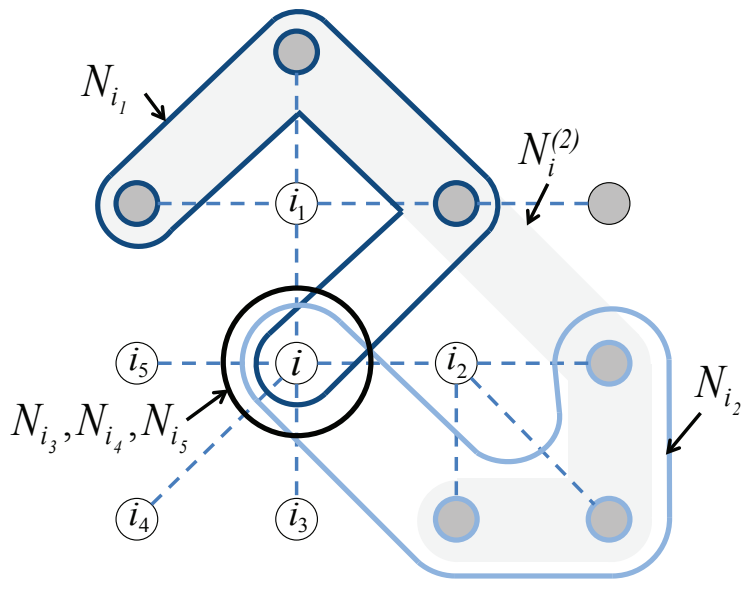

Figure 4: An example tree network. Notice the eliminated links from Fig. 1 to avoid multi path information diffusion.

as follows

$$
\begin{aligned}
\left\{y_{j, \tau}\right\}_{j \in \mathcal{N}_{i}^{(k)}}=\{ & \left\{y_{j, \tau}\right\}_{j \in \mathcal{N}_{i}^{(k)} \cap \mathcal{N}_{j_{1}}^{(k-1)}}, \\
& \left.\cdots,\left\{y_{j, \tau}\right\}_{j \in \mathcal{N}_{i}^{(k)} \cap \mathcal{N}_{j_{i}}^{(k-1)}}\right\} .
\end{aligned}
$$

In the time-stamped information disclosure, at time $t=3$, agent- $i$ has access to $\delta_{i, 3}^{o}$, defined in (2). We denote the set of new measurements received by $i$ over $j$ at time $t=2$ by

$$
\Delta_{j, i, 2}^{o}:=\{\overbrace{\left\{y_{k, 2}\right\}_{k \in \mathcal{N}_{i}^{(1)} \cap \mathcal{N}_{j}^{(0)}}}^{=y_{j, 2}},\left\{y_{k, 1}\right\}_{k \in \mathcal{N}_{i}^{(2)} \cap \mathcal{N}_{j}^{(1)}}\},
$$

which can also be written as

$$
\Delta_{j, i, 2}^{o}=\delta_{j, 2}^{o} \backslash\{\overbrace{y_{j, 1}}^{=\delta_{j, 1}^{o}}, \underbrace{y_{i, 1}}_{=\Delta_{i, j, 1}^{o}=\delta_{i, 1}^{o}}\},
$$

where we exclude the information sent by $i$ to $j$ at time $t=1$, i.e., $y_{i, 1}$. Then, we can write the accessed information as the union of new measurement $y_{i, 3}$, new measurements received over the neighboring agents and the accessed information at time $t=2$ as follows:

$$
\delta_{i, 3}^{o}=\left\{y_{i, 3}, \Delta_{j_{1}, i, 2}^{o}, \cdots, \Delta_{j_{i}, i, 2}^{o}, \delta_{i, 2}^{o}\right\}
$$


Note that the sets on the right hand side of $(16)$ are disjoint due to tree structure.

Furthermore, by (15) and (16), the sigma-algebra generated by $\delta_{i, 3}^{o}$ is equivalent

to the sigma-algebra generated by the set $\left\{\left\{y_{i, \tau}\right\}^{\tau \leq 3},\left\{\delta_{j, \tau}^{o}\right\}_{j \in \mathcal{N}_{i}}^{\tau \leq 2}\right\}$. Since $u_{j, t}^{o}=$ $E\left[\boldsymbol{x} \mid \boldsymbol{\delta}_{j, t}^{o}=\delta_{j, t}^{o}\right]$, we obtain

$$
u_{i, 3}^{o}=E\left[\boldsymbol{x} \mid\left\{\boldsymbol{y}_{i, \tau}=y_{i, \tau}\right\}^{\tau \leq 3},\left\{\boldsymbol{u}_{j, \tau}^{o}=u_{j, \tau}^{o}\right\}_{j \in \mathcal{N}_{i}}^{\tau \leq 2}\right] .
$$

By 15, we have

$$
\Delta_{j, i, t}^{o}=\delta_{j, t}^{o} \backslash\left\{\delta_{j, t-1}^{o} \cup \Delta_{i, j, t-1}^{o}\right\},
$$

250

which implies that for $t \geq 2, \Delta_{j, i, t}^{o}$ is constructible from $\delta_{i, \tau}^{o}$ and $\delta_{j, \tau}^{o}$ for $\tau \leq t$. Hence, by induction, we conclude that the lower bound can be achieved through the exchange of local estimates.

Remark 3.1: When the expectation of the state is conditioned on infinite number of observations over even a constructed spanning tree, only a finite number of the observations is missing compared to the case over a fully connected network. Hence, even if we construct the spanning tree of that network, we would still achieve the lower bound over a fully connected (or centralized) network asymptotically. As an illustrative example, in Fig. 10, we observe that the MMSE performance over the fully connected, star and line networks are asymptotically the same. Similarly, in [26, 27, 28], the authors show that the performance of the diffusion based algorithms could approach the performance of a fully connected network under certain regularity conditions.

In the sequel, we propose the OEDOL algorithm that achieves the lower bound over tree networks iteratively.

\subsection{OEDOL Algorithm}

By Theorem 3.1, over a tree network, oracle action can be constructed by

$$
u_{i, t}^{o}=E\left[\boldsymbol{x} \mid\left\{\boldsymbol{y}_{i, \tau}=y_{i, \tau}\right\}^{\tau \leq t},\left\{\boldsymbol{u}_{j, \tau}^{o}=u_{j, \tau}^{o}\right\}_{j \in \mathcal{N}_{i}}^{\tau \leq t-1}\right]
$$

through the disclosure of oracle actions, i.e., local estimates. We remark that $u_{i, t}^{o}$ is linear in the previous actions $u_{i, \tau}^{o}, \tau \leq t-1$. In order to extract new 
information, i.e., innovation part, we need to eliminate the previously received information at each instant on the neighboring agents. This brings in additional computational complexity. On the contrary, agents can just send the new information compared to the previously sent information, e.g., $s_{i, t}$. Note that here agents disclose the same information to the neighboring agents. Since we are conditioning on the linear combinations of the conditioned variables without effecting their spanned space, i.e., $s_{i, t}$ is computable from $u_{i, \tau}^{o}$ for $\tau \leq t$ and vice versa, agents can still achieve the oracle performance by reduced computational load, yet.

At time $t$, agent- $i$ receives local measurement $y_{i, t}$ and sent information from the neighboring agents, $r_{i, t}:=\operatorname{col}\left\{s_{j_{1}, t-1}, \cdots, s_{j_{\pi_{i}}, t-1}\right\}$. We aim to determine the content of the received information $r_{i, t}$ to extract the innovation within them and utilize this innovation in the update of the oracle action.

Initially, at time $t=1$, agent- $i$ has only access to the local measurement $y_{i, 1}$. Then, the oracle action is given by

$$
u_{i, 1}^{o}=\left(I-\Sigma_{x} H_{i}^{\prime}\left(H_{i} \Sigma_{x} H_{i}^{\prime}+\Sigma_{n_{i}}\right)^{-1} H_{i}\right) \bar{x}+\Sigma_{x} H_{i}^{\prime}\left(H_{i} \Sigma_{x} H_{i}^{\prime}+\Sigma_{n_{i}}\right)^{-1} y_{i, 1} .
$$

Let $u_{i, 0}^{o}=\bar{x}$ and $\hat{\Sigma}_{i, 0}=\Sigma_{x}$, and set $B_{i, 1}=\hat{\Sigma}_{i, 0} H_{i}^{\prime}\left(H_{i} \hat{\Sigma}_{i, 0} H_{i}^{\prime}+\Sigma_{n_{i}}\right)^{-1}$ and $A_{i, 1}=I-B_{i, 1} H_{i}$. Then, we obtain

$$
\begin{aligned}
& u_{i, 1}^{o}=A_{i, 1} u_{i, 0}^{o}+B_{i, 1} y_{i, 1}, \\
& \hat{\Sigma}_{i, 1}=A_{i, 1} \hat{\Sigma}_{i, 0} .
\end{aligned}
$$

Next, instead of sending $u_{i, 1}^{o}$, agent- $i$ sends to the neighboring agents, $j \in \mathcal{N}_{i}$,

$$
\begin{aligned}
s_{i, 1} & =u_{i, 1}^{o}-A_{i, 1} u_{i, 0}^{o} \\
& =B_{i, 1} y_{i, 1} .
\end{aligned}
$$

Correspondingly, at time $t=2$, agent- $i$ receives $y_{i, 2}$ and $r_{i, 2}$. Let $\boldsymbol{r}_{i, 2}$ be the corresponding random vector. Then, conditioning the state and the received information on the previously available information $\boldsymbol{y}_{i, 1}=y_{i, 1}$, we have

$$
\left[\begin{array}{c}
\boldsymbol{x} \\
\boldsymbol{y}_{i, 2} \\
\boldsymbol{r}_{i, 2}
\end{array}\right] \mid \boldsymbol{y}_{i, 1}=y_{i, 1} \sim \mathbb{N}\left(\left[\begin{array}{c}
u_{i, 1}^{o} \\
H_{i} u_{i, 1}^{o} \\
\bar{H}_{i, 1} u_{i, 1}^{o}
\end{array}\right],\left[\begin{array}{ccc}
\hat{\Sigma}_{i, 1} & \hat{\Sigma}_{i, 1} H_{i}^{\prime} & \hat{\Sigma}_{i, 1} \bar{H}_{i, 1}^{\prime} \\
H_{i} \hat{\Sigma}_{i, 1} & H_{i} \hat{\Sigma}_{i, 1} H_{i}^{\prime}+\Sigma_{n_{i}} & H_{i} \hat{\Sigma}_{i, 1} \bar{H}_{i, 1}^{\prime} \\
\bar{H}_{i, 1} \hat{\Sigma}_{i, 1} & \bar{H}_{i, 1} \hat{\Sigma}_{i, 1} H_{i}^{\prime} & \bar{H}_{i, 1} \hat{\Sigma}_{i, 1} \bar{H}_{i, 1}^{\prime}+\bar{G}_{i, 1}
\end{array}\right]\right),
$$


where $\bar{H}_{i, 1}:=\operatorname{col}\left\{B_{j_{1}, 1} H_{j_{1}}, \cdots, B_{j_{i}, 1} H_{j_{\pi_{i}}}\right\}$ and $\bar{G}_{i, 1}=\operatorname{diag}\left\{G_{i, 1}\right\}$, where $G_{i, 1}:=\operatorname{col}\left\{B_{j_{1}, 1} \Sigma_{n_{j_{1}}} B_{j_{1}, 1}^{\prime}, \cdots, B_{j_{i}, 1} \Sigma_{n_{j_{i}}} B_{j_{i}, 1}^{\prime}\right\}$.

Let $\tilde{H}_{i, 1}:=\operatorname{col}\left\{H_{i}, \bar{H}_{i, 1}\right\}$ and $\tilde{G}_{i, 1}:=\operatorname{diag}\left\{\Sigma_{n_{i}}, \bar{G}_{i, 1}\right\}$ and set

$$
\begin{aligned}
{\left[\begin{array}{ll}
B_{i, 2} & C_{i, 2}
\end{array}\right] } & =\hat{\Sigma}_{i, 1} \tilde{H}_{i, 1}^{\prime}\left(\tilde{H}_{i, 1} \hat{\Sigma}_{i, 1} \tilde{H}_{i, 1}^{\prime}+\tilde{G}_{i, 1}\right)^{-1}, \\
A_{i, 2} & =I-B_{i, 2} H_{i}-C_{i, 2} \bar{H}_{i, 1} .
\end{aligned}
$$

Then, we obtain

$$
\begin{aligned}
& u_{i, 2}^{o}=A_{i, 2} u_{i, 1}^{o}+B_{i, 2} y_{i, 2}+C_{i, 2} r_{i, 2} \\
& \hat{\Sigma}_{i, 2}=A_{i, 2} \hat{\Sigma}_{i, 1}
\end{aligned}
$$

and agent- $i$ sends

$$
\begin{aligned}
s_{i, 2} & =u_{i, 2}^{o}-A_{i, 2} u_{i, 1}^{o} \\
& =B_{i, 2} y_{i, 2}+\sum_{j \in \mathcal{N}_{i}} C_{i, 2}^{(j)} \underbrace{B_{j, 1} y_{j, 1}}_{=s_{j, 1}},
\end{aligned}
$$

where $C_{i, 2}^{(j)}$ denotes the corresponding $j$ th block of $C_{i, 2}$. Therefore, at time $t=3$, agent- $i$ receives from $j \in \mathcal{N}_{i}$ :

$$
s_{j, 2}=B_{j, 2} y_{j, 2}+\sum_{k \in \mathcal{N}_{j} \backslash i}\left(C_{j, 2}^{(k)} B_{j, 1} y_{j, 1}\right)+C_{j, 2}^{(i)} B_{i, 1} y_{i, 1} .
$$

Since the last term on the right hand side of 22 is known by $i$, we have

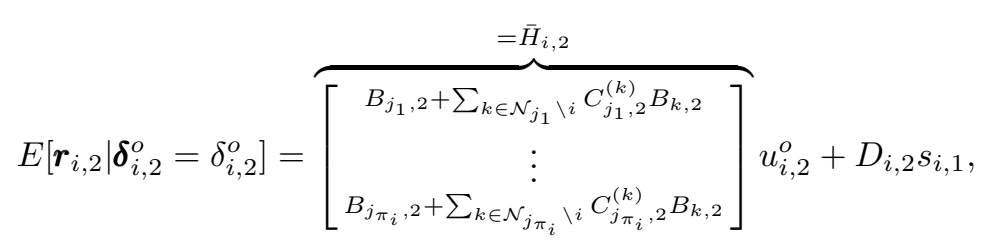

where $D_{i, 2}:=\operatorname{col}\left\{C_{j_{1}, 2}^{(i)}, \cdots, C_{j_{\pi_{i}}, 2}^{(i)}\right\}$, and

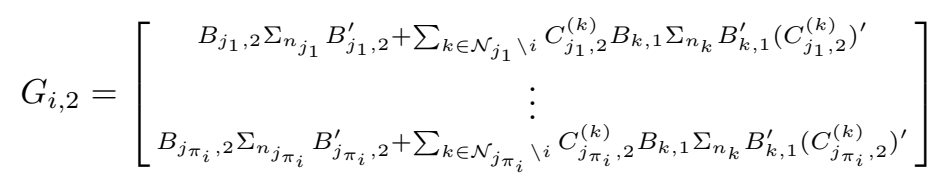

By (21), 23), and (24), the next oracle action $u_{i, 3}^{o}$ is given by

$$
u_{i, 3}^{o}=A_{i, 3} u_{i, 2}^{o}+B_{i, 3} y_{i, 3}+C_{i, 3}\left(r_{i, 3}-D_{i, 2} s_{i, 1}\right) .
$$


Subsequently, agent- $i$ sends $s_{i, 3}=u_{i, 3}^{o}-A_{i, 3} u_{i, 2}^{o}$ and the received information from $j \in \mathcal{N}_{i}$ yields

$$
\begin{aligned}
s_{j, 3}= & B_{j, 3} y_{j, 3}+\sum_{k \in \mathcal{N}_{j}} C_{j, 3}^{(k)}\left(B_{k, 2} y_{k, 2}+\sum_{l \in \mathcal{N}_{k} \backslash j} C_{k, 2}^{(l)} B_{l, 1} y_{l, 1}\right) \\
= & B_{j, 3} y_{j, 3}+\sum_{k \in \mathcal{N}_{j} \backslash i} C_{j, 3}^{(k)}\left(B_{k, 2} y_{k, 2}+\sum_{l \in \mathcal{N}_{k} \backslash j} C_{k, 2}^{(l)} B_{l, 1} y_{l, 1}\right) \\
& +C_{j, 3}^{(i)}\left(s_{i, 2}-C_{i, 2}^{(j)} s_{j, 1}\right) .
\end{aligned}
$$

Then, $\bar{H}_{i, 3}$ is given by

$$
\bar{H}_{i, 3}=\left[\begin{array}{c}
B_{j_{1}, 3} H_{j_{1}}+C_{j_{1}, 3} \bar{H}_{j_{1}, 2} \\
\vdots \\
B_{j_{\pi_{i}}, 3} H_{j_{\pi_{i}}}+C_{j_{\pi_{i}}, 3} \bar{H}_{j_{\pi_{i}}, 2}
\end{array}\right]-\left[\begin{array}{c}
C_{j_{1}, 3}^{(i)} \bar{H}_{j_{1}, 2}^{(i)} \\
\vdots \\
C_{j_{\pi_{i}}, 3}^{(i)} \bar{H}_{j_{\pi_{i}}, 2}^{(i)}
\end{array}\right]
$$

Correspondingly, we have

$$
G_{i, 3}=\left[\begin{array}{c}
B_{j_{1}, 3} \Sigma_{n_{j_{1}}} B_{j_{1}, 3}^{\prime}+C_{j_{1}, 3} \bar{G}_{j_{1}, 2} C_{j_{1}, 3}^{\prime} \\
\vdots \\
B_{j_{\pi_{i}}, 3} \Sigma_{n_{j_{i}}} B_{j_{\pi_{i}}, 3}^{\prime}+C_{j_{\pi_{i}}, 3} \bar{G}_{j_{\pi_{i}}, 2} C_{j_{\pi_{i}}, 3}^{\prime}
\end{array}\right]-\left[\begin{array}{c}
C_{j_{1}, 3}^{(i)} G_{j_{1}, 2}^{(i)}\left(C_{j_{1}, 3}^{(i)}\right)^{\prime} \\
\vdots \\
C_{j_{\pi_{i}, 3}(i)}^{(i)} G_{j_{\pi_{i}, 2},\left(C_{j_{\pi_{i}}, 3}\right)^{\prime}}^{(i)}
\end{array}\right] .
$$

Therefore, the oracle action can be written as

$$
u_{i, 4}^{o}=A_{i, 4} u_{i, 3}^{o}+B_{i, 4} y_{i, 4}+C_{i, 4}\left(r_{i, 4}-D_{i, 3} s_{i, 2}+T_{i, 3} r_{i, 2}\right),
$$

where $A_{i, 4}, B_{i, 4}, C_{i, 4}, D_{i, 3}$ are defined accordingly and

$$
T_{i, 3}:=\left[\begin{array}{lll}
C_{j_{1}, 3}^{(i)} C_{i, 2}^{\left(j_{1}\right)} & & \\
& \ddots & \\
& & C_{j_{i}, 3}^{(i)} C_{i, 2}^{\left(j \pi_{i}\right)}
\end{array}\right]
$$

Following identical steps, for $t \geq 1$, the OEDOL algorithm is given by

$$
\begin{aligned}
& u_{i, t}^{o}=A_{i, t} u_{i, t-1}^{o}+B_{i, t} y_{i, t}+C_{i, t} w_{i, t}, \\
& \hat{\Sigma}_{i, t}=A_{i, t} \hat{\Sigma}_{i, t-1},
\end{aligned}
$$

where $w_{i, t}$ is the innovation term extracted from the received information, which evolves according to

$$
w_{i, t}=r_{i, t}-D_{i, t-1} s_{i, t-2}+T_{i, t-1} w_{i, t-2} .
$$


The weighting matrices $A_{i, t}, B_{i, t}, C_{i, t}, D_{i, t}$, and $T_{i, t}$ are defined by

$$
\begin{gathered}
{\left[\begin{array}{ll}
B_{i, t} & C_{i, t}
\end{array}\right]=\hat{\Sigma}_{i, t-1} \tilde{H}_{i, t-1}^{T} \times} \\
\left(\tilde{H}_{i, t-1} \hat{\Sigma}_{i, t-1} \tilde{H}_{i, t-1}^{T}+\tilde{G}_{i, t-1}\right)^{-1}, \\
A_{i, t}=I-B_{i, t} H_{i}-C_{i, t} \bar{H}_{i, t-1}, \\
D_{i, t}=\operatorname{col}\left\{C_{j_{1}, t}^{(i)}, \cdots, C_{j_{\pi_{i}}, t}^{(i)}\right\} \\
T_{i, t}=\left[\begin{array}{ccc}
C_{j_{1}, t}^{(i)} C_{i, t-1}^{\left(j_{1}\right)} & \cdots & \mathbf{0} \\
\vdots & \ddots & \vdots \\
\mathbf{0} & \cdots & C_{j_{\pi_{i}, t}^{(i)}}^{(i)} C_{i, t-1}^{\left(j_{\pi_{i}}\right)}
\end{array}\right] .
\end{gathered}
$$

where $\tilde{H}_{i, t}=\operatorname{col}\left\{H_{i}, \bar{H}_{i, t}\right\}, \tilde{G}_{i, t}=\operatorname{diag}\left\{\Sigma_{n_{i}}, \bar{G}_{i, t}\right\}$ and $\bar{G}_{i, t}=\operatorname{diag}\left\{G_{i, t}\right\}$. By (25) and 26), the intermediate parameters $\bar{H}_{i, t}$ and $G_{i, t}$ evolve according to

$$
\begin{aligned}
\bar{H}_{i, t}= & {\left[\begin{array}{c}
B_{j_{1}, t} H_{j_{1}}+C_{j_{1}, t} \bar{H}_{j_{1}, t-1} \\
\vdots \\
B_{j_{\pi_{i}}, t} H_{j_{\pi_{i}}}+\dot{C}_{j_{\pi_{i}}, t} \bar{H}_{j_{\pi_{i}}, t-1}
\end{array}\right]-\left[\begin{array}{c}
C_{j_{1}, t}^{(i)} \bar{H}_{j_{1}, t-1}^{(i)} \\
\vdots \\
C_{j_{\pi_{i}}, t}^{(i)} \\
G_{i, t}^{(i)}
\end{array}=\left[\begin{array}{c}
B_{j_{\pi_{i}}, t}, t-1 \\
\Sigma_{n_{j_{1}}} B_{j_{1}, t}^{T}+C_{j_{1}, t} \bar{G}_{j_{1}, t-1} C_{j_{1}, t}^{T} \\
\vdots \\
B_{j_{\pi_{i}}, t} \Sigma_{n_{j_{\pi_{i}}}} B_{j_{\pi_{i}, t}}^{T}+C_{j_{\pi_{i}}, t} \bar{G}_{j_{\pi_{i}}, t-1} C_{j_{\pi_{i}}, t}^{T}
\end{array}\right]-\left[\begin{array}{c}
C_{j_{1}, t}^{(i)} G_{j_{1}, t-1}^{(i)}\left(C_{j_{1}, t}^{(i)}\right)^{T} \\
\vdots \\
C_{j_{\pi_{i}, t}}^{(i)} G_{j_{\pi_{i}}, t-1}^{(i)}\left(C_{j_{\pi_{i}}, t}^{(i)}\right)^{T}
\end{array}\right]\right.}
\end{aligned}
$$

and we initialize the parameters as $\bar{H}_{j, \tau}=\mathbf{0}$ and $G_{j, \tau}=\mathbf{0}$ for $\tau<1$.Then, agent- $i$ sends

$$
s_{i, t}=u_{i, t}^{o}-A_{i, t} u_{i, t-1}^{o} .
$$

The detailed description of the algorithm is provided in Table 1.

\subsection{Computational Complexity}

In (27), the combination matrices $A_{i, t}, B_{i, t}, C_{i, t}, D_{i, t-1}$, and $T_{i, t-1}$ are independent of the streaming data although they are time-varying. Hence they can be computed before-hand. In that case, the computational complexity of the iterations for each agent is dominated by the term $C_{i, t} w_{i, t}$. Therefore, the average computational complexity is on the order of $p^{2} \pi^{2}$, where $\pi^{2}:=1 / m \sum_{i=1}^{m} \pi_{i}^{2}$, i.e., $O\left(p^{2} \pi^{2}\right)$. Otherwise, the computational complexity of the algorithm is mainly dominated by the matrix inversion in $[30)$, note that $\bar{G}_{i, t} \in \mathbb{R}^{p \pi_{i} \times p \pi_{i}}$, unless the network is sparsely connected, i.e., $\pi_{i} \ll m$ for $i=1, \cdots, m$. Therefore, 
Table 1: The description of the OEDOL algorithm.

Algorithm: The OEDOL Algorithm

\section{Initialization:}

For $i=1$ to $m$ do

$$
\begin{aligned}
& u_{i, 0}^{o}=\bar{x}, \hat{\Sigma}_{i, 0}=\Sigma_{x}, \\
& \bar{H}_{i, \tau}=\mathbf{0}, G_{i, \tau}=\mathbf{0}, \text { and } w_{i, \tau}=\mathbf{0} \text { for } \tau<1
\end{aligned}
$$

End for

\section{Iterations:}

Do for $t \geq 1$

For $i=1$ to $m$ do

\section{Construction of Weights:}

For $j=1$ to $m$ do

Calculate $\bar{H}_{j, t}$ and $G_{j, t}$ by (34) and (35).

Determine combination matrices via (30), (31), 32), and (33).

End for

Construct $r_{i, t}$ through received $s_{j, t-1}$ for $j \in \mathcal{N}_{i}$

Extraction of Innovation:

$w_{i, t}=r_{i, t}-D_{i, t-1} s_{i, t-2}+T_{i, t-1} w_{i, t-2}$

Update:

$u_{i, t}^{o}=A_{i, t} u_{i, t-1}^{o}+B_{i, t} y_{i, t}+C_{i, t} w_{i, t}$

$\hat{\Sigma}_{i, t}=A_{i, t} \hat{\Sigma}_{i, t-1}$

Disclose $s_{i, t}=u_{i, t}^{o}-A_{i, t} u_{i, t-1}^{o}$ to the neighbors.

End for 
Table 2: A comparison of the computational complexities of the proposed algorithms.

\begin{tabular}{|c|c|c|}
\hline Algorithm & Without weights & Pre-computed weights \\
\hline ODOL algorithm & $O\left((q m)^{3}\right)$ & $O\left((q m)^{2}\right)$ \\
\hline OEDOL algorithm & $O\left(m p^{3} \pi^{3}\right)$ & $O\left(p^{2} \pi^{2}\right)$ \\
\hline
\end{tabular}

over a non-sparse network, the average complexity is on the order of $m p^{3} \pi^{3}\left(\pi^{3}\right.$ tree structure.

\subsection{Tree Networks Involving Cell Structures}

While constructing the spanning tree, we cancel certain communication links in order to avoid multi-path information propagation. However, we also observe

that in a fully connected network agents can achieve the oracle performance through the disclosure of local observations. In particular, since all of the agents 
are connected, each agent can receive the observations across the network directly. Correspondingly, in a fully connected network, we can achieve identical performance with the ODOL algorithm only through the disclosure of the local estimates as stated in the following corollary formally.

Corollary 3.1: Consider the team-problem over a fully connected network, in which $r=p$. Then, exchange of local estimates can lead to the team-optimal solution, i.e., agents can achieve the oracle performance.

Proof: Over a fully connected network, $\kappa_{i}=1$ and the oracle action is given 340 by

$$
u_{i, t}^{o}=E\left[\boldsymbol{x} \mid\left\{\boldsymbol{y}_{i, \tau}=y_{i, \tau}\right\}^{\tau \leq t},\left\{\boldsymbol{y}_{j, \tau}=\mathbf{y}_{j, \tau}\right\}_{j \in \mathcal{N}_{i}}^{\tau \leq t-1}\right]
$$

and we can also obtain (36) by (11) since we have

$$
\Delta_{j, i, t}^{o}=\delta_{j, t}^{o} \backslash\left\{\delta_{i, t}^{o} \backslash\left\{y_{i, t}\right\}\right\}
$$

which implies that $\Delta_{j, i, t}^{o}$ is constructible from $\delta_{i, \tau}^{o}$ and $\delta_{j, \tau}^{o}$ for $\tau \leq t$. The proof is concluded.

We point out that the team-optimal strategies can fail if a link or node failure occurs. However, once a link failure is detected, team-optimal strategies can be recomputed by eliminating the failed link in the new network configuration. Hence, through such strategies, we can increase the robustness of the team against link and node failures.

We define a "cell structure" as a sub-network in which all agents are connected to each other. Intuitively, considering a cell structure as a "single" agent, the cell (i.e., all the agents in the cell) can be involved in the tree such that the agents can still achieve the oracle performance through the disclosure of local estimates (although there may be loops in the cell). We list the features of the cell structures, e.g., seen in Fig. 5, as follows:

- Agents out of a cell can connect to at most one of the agents within that cell.

- A cell structure consists of at least 2 agents. 


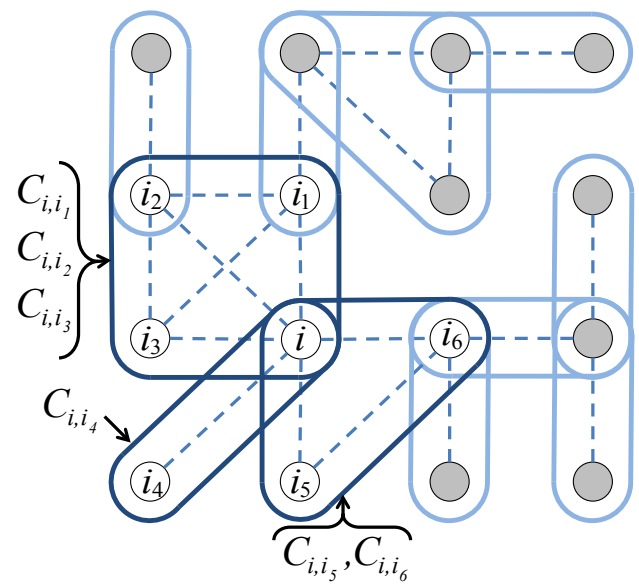

Figure 5: An example tree network involving cell structures.

- An agent can belong to more than one cell.

- Two different agents cannot belong to more than one cell at the same time.

- All of the agents belong to at least a cell in a connected network.

- Each agent has also the knowledge of the cells of the other agents.

- Each agent labels its cells from its own and its first order neighbor's point of view. As an example, for agent- $i, \mathcal{C}_{i, i_{1}}$ denotes the cell involving both $i$ and $i_{1}$. Note that if the same cell also includes $i_{2}, \mathcal{C}_{i, i_{1}}=\mathcal{C}_{i, i_{2}}$.

The following theorem shows that agents can achieve the oracle performance over tree networks involving cell structures through the disclosure of the local estimates.

Theorem 3.2: Consider the team problem over tree networks involving cell structures. Then, exchange of local estimates can lead to the team-optimal solution, i.e., agents can achieve the oracle performance.

Proof: Initially, we have $\Delta_{j, i, 1}^{o}=\delta_{j, 1}=\left\{y_{j, 1}\right\}$ and the oracle action is also given by (11) over this network topology. Note that the information received 
by $j$ at $t=2$ is given by $\delta_{j, 2}^{o}=\left\{y_{j, 2},\{\overbrace{y_{k, 1}}^{=\Delta_{k, j, 0}^{o}}\}_{k \in \mathcal{N}_{j}}, \delta_{j, 1}^{o}\right\}$, which yields

$$
\begin{aligned}
\Delta_{j, i, 2}^{o} & =\delta_{j, 2}^{o} \backslash\left\{\delta_{j, 1}^{o} \cup \bigcup_{k \in \mathcal{C}_{i, j}}\left\{y_{k, 1}\right\}\right\}, \\
& =\delta_{j, 2}^{o} \backslash\left\{\delta_{j, 1}^{o} \cup \bigcup_{k \in \mathcal{C}_{i, j}} \Delta_{k, j, 1}^{o}\right\}
\end{aligned}
$$

and $\Delta_{j, j, t}^{o}=\varnothing$ by definition. Due to the cell structure, we have

$$
\begin{aligned}
\bigcup_{k \in \mathcal{C}_{j, i}} \Delta_{k, j, 1}^{o} & =\Delta_{i, j, 1}^{o} \cup \underset{k \in \mathcal{C}_{i, j} \backslash j}{\bigcup} \Delta_{k, i, 1}^{o}, \\
& =\delta_{i, 1}^{o} \cup \bigcup_{k \in \mathcal{C}_{i, j} \backslash j} \delta_{k, 1}^{o} .
\end{aligned}
$$

Correspondingly, for $t>0$ we have

$$
\Delta_{j, i, t}^{o}=\delta_{j, t}^{o} \backslash\left\{\delta_{j, t-1}^{o} \cup \Delta_{i, j, t-1}^{o} \cup \bigcup_{k \in \mathcal{C}_{i, j} \backslash j} \Delta_{k, i, t-1}^{o}\right\}
$$

and $\Delta_{j, i, t}^{o}$ is constructible by the sets $\delta_{i, \tau}^{o}$ and $\delta_{j, \tau}^{o}$ for $j \in \mathcal{N}_{i}$ and $\tau \leq t$. Note that over tree networks, $\mathcal{C}_{i, j} \backslash j=\varnothing$ for $i=1, \cdots, m, j \in \mathcal{N}_{i}$, and (37) leads to 18. Hence, for $t>0$ we obtain (19) and the proof is concluded.

Note that the network can have loops within the cell structures and agents can still achieve the oracle performance through the diffusion of the local estimates. This increases the robustness of the team strategies against the link failures. In the sequel, we provide the sub-optimal extensions of the algorithms for practical applications.

\section{Sub-optimal Approaches}

Minimization of the cost function (4) optimally requires relatively excessive computations. We aim to mitigate the problem sub-optimally yet in a computationally efficient approach while achieving comparable performance with the optimal case. As an example, we can approximate the cost measure (4) through 


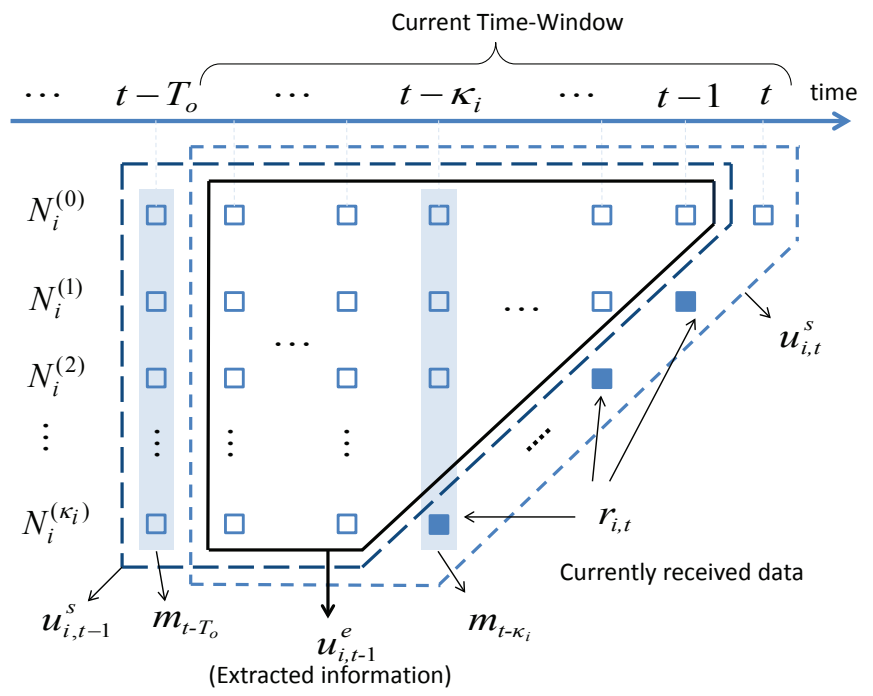

Figure 6: Information aggregation illustration. Small squares represent the information across the agents in the corresponding neighborhood and time.

$T_{o} \geq \max \left\{\kappa_{i}\right\}_{i=1, \cdots, m}$ size time-windowing as follows

$$
\sum_{t=1}^{T} \min _{\eta_{i, t} \in \Omega_{i, t}^{s}} E\left\|\boldsymbol{x}-\eta_{i, t}\left(\delta_{i, t}^{s}\right)\right\|^{2},
$$

where agent- $i$ has the information set

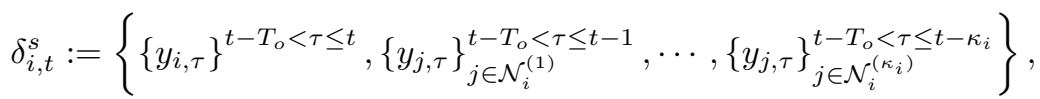

if $t \geq T_{o}$ and other cases are defined accordingly, $\sigma_{i, t}^{s}$ is the sigma-algebra generated by $\delta_{i, t}^{s}$, and $\Omega_{i, t}^{o}$ denotes the set of all $\sigma_{i, t}^{s}$-measurable functions from $\mathbb{R}^{q \kappa} \times \mathbb{R}^{q \pi_{i}(\kappa-1)} \times \cdots \times \mathbb{R}^{q \pi_{i}^{\kappa_{i}\left(\kappa-\kappa_{i}\right)}}$ to $\mathbb{R}^{p}$. Then, team-optimal action is given by

$$
u_{i, t}^{s}=E\left[\boldsymbol{x} \mid \boldsymbol{\delta}_{i, t}^{s}=\delta_{i, t}^{s}\right],
$$

where $\boldsymbol{\delta}_{i, t}^{s}$ is the set of corresponding random parameters.

In Fig. 6, we illustrate the time-windowing approach for time stamped information disclosure. We define a memory element $m_{t}$ denoting the expectation of the state conditioned on all measurement at time $t$, i.e., $m_{t}:=E\left[\boldsymbol{x} \mid\left\{\boldsymbol{y}_{i, t}=\right.\right.$ 
$\left.\left.y_{i, t}\right\}_{i=1}^{m}\right]$. We consider that $\bar{x}=\mathbf{0}$. Then, we can write $m_{t}=M y_{t}$, where $M:=\Sigma_{x} H^{\prime}\left(H \Sigma_{x} H^{\prime}+\Sigma_{n}\right)^{-1}$ and $y_{t}:=\operatorname{col}\left\{y_{1, t}, \cdots, y_{m, t}\right\}$.

Let $u_{i, t-1}^{e}$ represent the extracted information from the estimate $u_{i, t-1}^{s}$ via the memory element $m_{t-T_{o}}$, i.e.,

$$
u_{i, t-1}^{e}:=E\left[\boldsymbol{x} \mid\left\{\boldsymbol{y}_{i, \tau}=y_{i, \tau}\right\}^{t-T_{o}+1<\tau \leq t-1}, \cdots,\left\{\boldsymbol{y}_{j, \tau}=y_{j, \tau}\right\}_{j \in \mathcal{N}_{i}^{\left(\kappa_{i}\right)}}^{t-T_{o}+1<\tau \leq t-\kappa_{i}-1}\right]
$$

if $t \geq T_{o}$ and other cases are defined accordingly. Then, we can write $u_{i, t-1}^{e}=$ $N_{i} y_{i, t-1}^{e}$, where $N_{i}=\Sigma_{x} \hat{H}_{i}^{\prime}\left(\hat{H}_{i} \Sigma_{x} \hat{H}_{i}^{\prime}+\hat{\Sigma}_{n_{i}}\right)^{-1}$ and $y_{i, t-1}^{e}$ is the vector collecting the measurements within that window (See Fig. 6). Additionally, we define

$$
\hat{H}_{i}:=\operatorname{col}\left\{H_{i}, H_{i}, H_{i}^{(1)}, H_{i}, H_{i}^{(1)}, H_{i}^{(2)}, \cdots, H_{i}, H_{i}^{(1)}, \cdots, H_{i}^{\left(\kappa_{i}\right)}\right\}
$$

and $H_{i}^{(k)}:=\operatorname{col}\left\{H_{j}\right\}_{j \in \mathcal{N}_{i}^{(k)}}$. Correspondingly, we have

$$
\hat{\Sigma}_{n_{i}}:=\operatorname{diag}\left\{\Sigma_{n_{i}}, \Sigma_{n_{i}}, \Sigma_{n_{i}}^{(1)}, \Sigma_{n_{i}}, \Sigma_{n_{i}}^{(1)}, \Sigma_{n, i}^{(2)}, \cdots, \Sigma_{n_{i}}, \Sigma_{n_{i}}^{(1)}, \cdots, \Sigma_{n_{i}}^{\left(\kappa_{i}\right)}\right\}
$$

and $\Sigma_{n_{i}}^{(k)}:=\operatorname{diag}\left\{\Sigma_{n_{j}}\right\}_{j \in \mathcal{N}_{i}^{(k)}}$. Therefore, for $t \geq T_{o}$, we have

$$
u_{i, t-1}^{s}=E\left[\boldsymbol{x} \mid \boldsymbol{u}_{i, t-1}^{e}=u_{i, t-1}^{e}, \boldsymbol{m}_{t-T_{o}}=m_{t-T_{o}}\right] .
$$

Note that $E\left[\boldsymbol{x} \mid \boldsymbol{m}_{t-T_{o}}=m_{t-T_{o}}\right]=m_{t-T_{o}}$ and $\hat{\Sigma}:=E\left[\left(\boldsymbol{x}-m_{t-T_{o}}\right)\left(\boldsymbol{x}-m_{t-T_{o}}\right)^{\prime}\right]=$ $\Sigma_{x} H^{\prime}\left(H \Sigma H^{\prime}+\Sigma_{n}\right)^{-1} H \Sigma=(I-M H) \Sigma$. Then, the distribution of $\boldsymbol{x}$ and $\boldsymbol{u}_{i, t-1}^{e}$ conditioned on $\boldsymbol{m}_{t-T_{o}}=m_{t-T_{o}}$ is given by

$$
\left[\begin{array}{c}
\boldsymbol{\boldsymbol { u } _ { i , t - 1 } ^ { e }} \\
\boldsymbol{\boldsymbol { x }}
\end{array}\right] \mid \boldsymbol{m}_{t-T_{o}}=m_{t-T_{o}} \sim \mathbb{N}\left(\left[\begin{array}{c}
m_{t-T_{o}} \\
N_{i} \hat{H}_{i} m_{t-T_{o}}
\end{array}\right],\left[\begin{array}{cc}
\hat{\Sigma} & \hat{\Sigma} \hat{H}_{i}^{\prime} N_{i}^{\prime} \\
N_{i} \hat{H}_{i} \hat{\Sigma} & N_{i} \hat{H}_{i} \hat{\Sigma} \hat{H}_{i}^{\prime} N_{i}^{\prime}+N_{i} \hat{\Sigma}_{n_{i}} N_{i}^{\prime}
\end{array}\right]\right)
$$

and we obtain

$$
\begin{aligned}
u_{i, t-1}^{s} & =m_{t-T_{o}}+\hat{\Sigma} \hat{H}_{i}^{\prime} N_{i}^{\prime}\left(N_{i} \hat{H}_{i} \hat{\Sigma} \hat{H}_{i}^{\prime} N_{i}^{\prime}+N_{i} \hat{\Sigma}_{n_{i}} N_{i}^{\prime}\right)^{-1}\left(u_{i, t-1}^{e}-N_{i} H_{i} m_{t-T_{o}}\right) \\
& =K_{i} m_{t-T_{o}}+L_{i} u_{i, t-1}^{e},
\end{aligned}
$$

where

$$
\begin{aligned}
L_{i} & :=\hat{\Sigma} \hat{H}_{i}^{\prime} N_{i}^{\prime}\left(N_{i} \hat{H}_{i} \hat{\Sigma} \hat{H}_{i}^{\prime} N_{i}^{\prime}+N_{i} \hat{\Sigma}_{n_{i}} N_{i}^{\prime}\right)^{-1}, \\
K_{i} & :=I-L_{i} N_{i} \hat{H}_{i} .
\end{aligned}
$$


Next, we aim to compute $u_{i, t}^{s}$ through $u_{i, t-1}^{s}$ and $\delta_{i, t}^{s}=\left\{y_{i, t-1}^{e}, y_{i, t}, r_{i, t}\right\}$, where $r_{i, t}$ is a vector consisting of currently received measurements as seen in Fig. 6. Let $\bar{H}_{i}:=\operatorname{col}\left\{H_{i}, H_{i}^{(1)}, \cdots, H_{i}^{\left(\kappa_{i}\right)}\right\}$ and $\bar{\Sigma}_{i}:=\operatorname{col}\left\{\Sigma_{n_{i}}, \Sigma_{n_{i}}^{(1)}, \cdots, \Sigma_{n_{i}}^{\left(\kappa_{i}\right)}\right\}$. ${ }_{410}$ Since $E\left[\left(\boldsymbol{x}-u_{i, t-1}^{e}\right)\left(\boldsymbol{x}-u_{i, t-1}^{e}\right)^{\prime}\right]=\Sigma_{x} \hat{H}_{i}^{\prime}\left(\hat{H}_{i} \Sigma_{x} \hat{H}_{i}^{\prime}+\hat{\Sigma}_{n}\right)^{-1} \hat{H}_{i} \Sigma_{x}=\left(I-N_{i} \hat{H}_{i}\right) \Sigma_{x}$, we have

$$
u_{i, t}^{s}=u_{i, t-1}^{e}+\Sigma_{x, i} \bar{H}_{i}^{\prime}\left(\bar{H}_{i} \Sigma_{x, i} \bar{H}_{i}^{\prime}+\bar{\Sigma}_{n_{i}}\right)^{-1}\left(\left[y_{i_{i, t}} r_{i, t}\right]-\bar{H}_{i} u_{i, t-1}^{e}\right),
$$

where $\Sigma_{x, i}:=\left(I-N_{i} \bar{H}_{i}\right) \Sigma_{x}$. By 40], $u_{i, t-1}^{e}=L_{i}^{-1}\left(u_{i, t-1}^{s}-K_{i} m_{t-T_{o}}\right)$, and we define the combination matrices

$$
\begin{aligned}
& {\left[\begin{array}{ll}
B_{i} & C_{i}
\end{array}\right]=\Sigma_{x, i} \bar{H}_{i}^{T}\left(\bar{H}_{i} \Sigma_{x, i} \bar{H}_{i}^{T}+\bar{\Sigma}_{n_{i}}\right)^{-1},} \\
& A_{i}:=\left(I-\left[\begin{array}{ll}
B_{i} & C_{i}
\end{array}\right] \bar{H}_{i}\right) L_{i}^{-1}, \\
& D_{i}:=A_{i} K_{i} M \text {. }
\end{aligned}
$$

Therefore, for $t \geq T_{o}$, the sub-optimal distributed online learning (SDOL) algorithm is given by

$$
u_{i, t}^{s}=A_{i} u_{i, t-1}^{s}+B_{i} y_{i, t}+C_{i} r_{i, t}-D_{i} y_{t-T_{o}} .
$$

In the following we provide several remarks about the practical implementation of the SDOL algorithm.

\section{Remark 4.1:}

- We point out that in the update (44) the matrices $A_{i}, B_{i}, C_{i}$, and $D_{i}$ are independent from the data and they are time invariant for $t \geq T_{o}$. Hence, they can be pre-computed and installed onto the agents. Then, the SDOL algorithm basically takes the linear average of the previous estimate, the current measurement and received data, and measurements at time $t-T_{o}$.

- Different from the conventional approaches, the SDOL algorithm requires to memorize previous observations. Note that if $q<p / m$, storing measurements individually rather than a linear combination, i.e., $m_{t}$, might be more efficient in terms of memory usage. 
- The computational complexity of the SDOL algorithm is $O\left(p^{2}+2 p m q\right)$ (or say $O\left(p^{2}\right)$ for $q \ll p / m$ ) due to matrix-vector multiplications in 44,, and the algorithm requires disclosure of a data vector with $(m-1) \times q$ dimensions.

- Finally, even though agent-i uses update (44) for $t<T_{o}$ assuming that $y_{j, \tau}=0$ for $j=1, \cdots, m$ and $\tau<1$, we have

$$
\begin{aligned}
E\left[\left(x-u_{i, \tau}^{s}\right)^{\prime}\left(x-u_{i, \tau}^{s}\right)\right] & =\Sigma_{x, i}-\Sigma_{x, i} \bar{H}_{i}^{T}\left(\bar{H}_{i} \Sigma_{x, i} \bar{H}_{i}^{T}+\bar{\Sigma}_{n_{i}}\right)^{-1} \bar{H}_{i} \Sigma_{x, i} \\
& =A_{i} L_{i} \Sigma_{x, i}
\end{aligned}
$$

for $\tau \geq T_{o}$ since at each iteration, agent-i excludes the impact of $y_{t-T_{o}}$ on $u_{i, t}^{s}$. This sharp change results in as a breaking point on the learning curve as seen in Fig. 15.

In Table 3, we tabulate a detailed description of the SDOL algorithm. Note that SDOL algorithm is based on the aggregation of information. Correspondingly, we can apply the time-windowing approach to the disclosure of local estimates and formulate a sub-optimal efficient distributed online learning algorithm (SEDOL) over tree networks.

In the sequel, we also provide illustrative simulations showing the enhanced tracking performance due to the proposed algorithm over several distributed network scenarios.

\section{Illustrative Examples}

In this section, we examine the performance of team optimal actions under different scenarios through numerical examples. To this end, we consider three main network structures, namely fully connected, star, and line networks with $m=20$ agents, and a randomly generated network seen in Fig. 7. In a fully connected network, each agent has a connection with each other and any new

\footnotetext{
${ }^{3}$ The action is no more team-optimal because of the assumption.
} 
Table 3: The description of the SDOL- $T_{o}$ algorithm.

Algorithm: The SDOL- $T_{o}$ algorithm

\section{Initialization:}

$u_{i, 0}^{s}=\mathbf{0}$ for all $i$

$y_{i, \tau}=\mathbf{0}$ for $\tau<1$ and all $i$

Calculate combination matrices via (41), (42) and (43).

\section{Update:}

Do for $t \geq 1$

For $i=1$ to $m$ do

Construct $r_{i, t}$ through received $s_{j, t-1}$ for $j \in \mathcal{N}_{i}$.

Store $r_{i, t}$ and $y_{i, t}$ in the memory accordingly.

$u_{i, t}^{s}=A_{i} u_{i, t-1}^{s}+B_{i} y_{i, t}+C_{i} r_{i, t}-D_{i} y_{t-T_{o}}$.

Erase $y_{t-T_{o}}$ from the memory.

Diffuse $s_{i, t}$ to the neighboring agents.

End for

measurement can be collected at each other after just one hop. This is the best possible connection such that the corresponding achievable team cost is the smallest. In a star network, there exists a pseudo-centralized agent, e.g., agent$1 \mathrm{in} \mathrm{Fig.} \mathrm{7b}$, and all the other agents are connected to that agent. Therefore, the furthest distance between any two agents is 2, e.g., there exist 2-hops between agent-2 and agent-3 (agent-2 to agent-1 then agent-1 to agent-3) in Fig. 7b. We have generated the network in Fig. $7 \mathrm{c}$ randomly such that number of neighbors of agent- $i, \pi_{i}$, is chosen uniformly between 2 to 10 . Therefore, the generated network can have loops, i.e., may not be a tree. Furthermore, in a line network, the connections between the agents form a line as seen in Fig. 7d. Contrary 


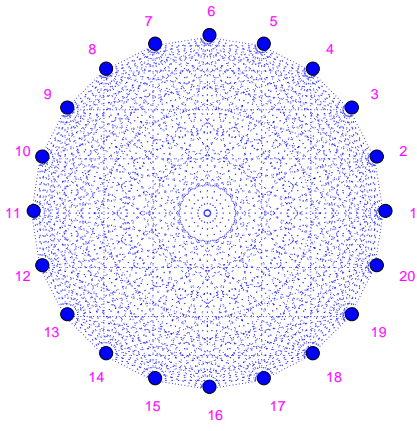

(a) Fully connected network

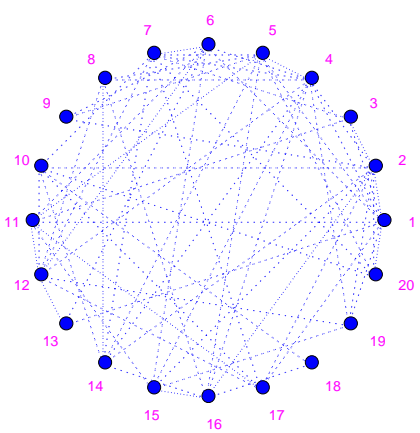

(c) Arbitrary network

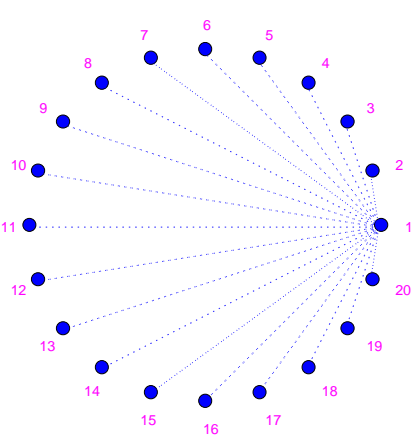

(b) Star network

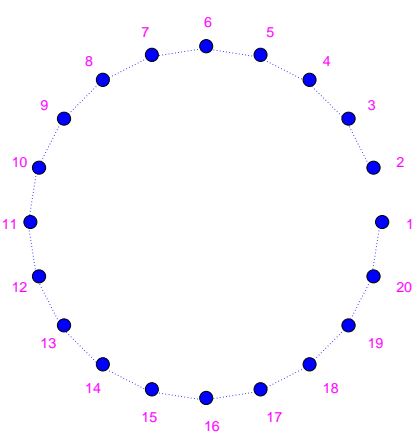

(d) Line network

Figure 7: Different network configurations.

to the fully connected network, a line network has the least possible connection such that any two agents are connected via a certain number of hops. Note that there exist $m-1=19$ hops between agent-1 and agent-2 in Fig. $7 \mathrm{~d}$

We analyze the performance of a team of agents over those network structures. We set $p=10, q=1, \bar{x}=\mathbf{0}, \Sigma_{x}=I$, and the standard deviation of the zero-mean measurement noise is chosen randomly from a folded normal distribution as seen in Fig. 8, Furthermore, we choose $H_{i}$ randomly from normal distribution, $\mathbb{N}(\mathbf{0}, I)$. In order to observe the change of oracle cost $(r \rightarrow \infty)$ for 


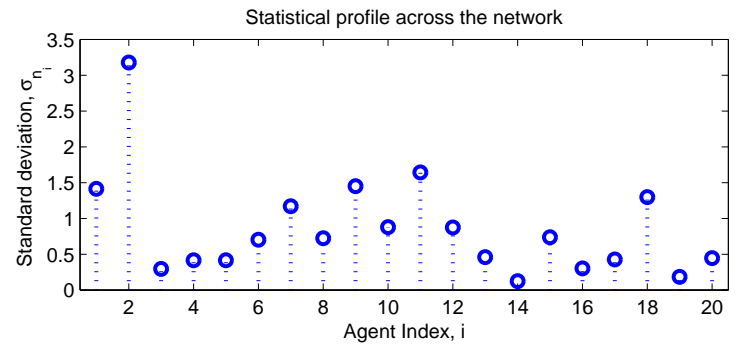

Figure 8: Standard deviation of measurement noise across the network.

different time horizons, we introduce

$$
J(T)=\min _{\substack{\eta_{i, t} \in \Omega_{i, t}, \ldots, T \\ i=1, \cdots, m, t=1, \cdots, T}} \sum_{t=1}^{T} \sum_{i=1}^{m} E\left\|\boldsymbol{x}-\eta_{i, t}\left(\delta_{i, t}^{o}\right)\right\|^{2},
$$

which is a function of the length of time horizon, $T$. We re-emphasize that in (46), each action at $t=1, \cdots, T$ has a cumulative impact on the cost. Let $\eta_{i, t}^{o}$, for $i=1, \cdots, m$ and $t=1, \cdots, T$, be the oracle strategies for $J(T)$. Then, we introduce a terminal cost function defined by

$$
P(T)=\sum_{i=1}^{m} E\left\|\boldsymbol{x}-\eta_{i, T}^{o}\left(\delta_{i, T}^{o}\right)\right\|^{2},
$$

which is the impact of final action on $J(T)$.

In Fig. 9, we compare $J(T)$, for $T=1, \cdots, 20$, over the fully connected, star, arbitrary, and line networks in Fig. 7 by ensemble averaging empirical cost of the ODOL algorithm over 100 independent trials. Note that if the associated network is a tree network, e.g., the star network is a tree, the same cost could be attained through the OEDOL algorithm as shown analytically in Section 3. We point out that Fig. 9 is not a time evolution of a learning algorithm. In particular, for each time horizon $T$, we compare the corresponding oracle cost over certain networks. As pointed out before, because of the most possible connection between the agents, the fully connected network results in the least oracle cost for each time horizon $T$ while the line network possesses the largest cost. Additionally, because of the cumulation of the impact of the actions at each time, for larger time horizon the corresponding oracle cost is also larger, 


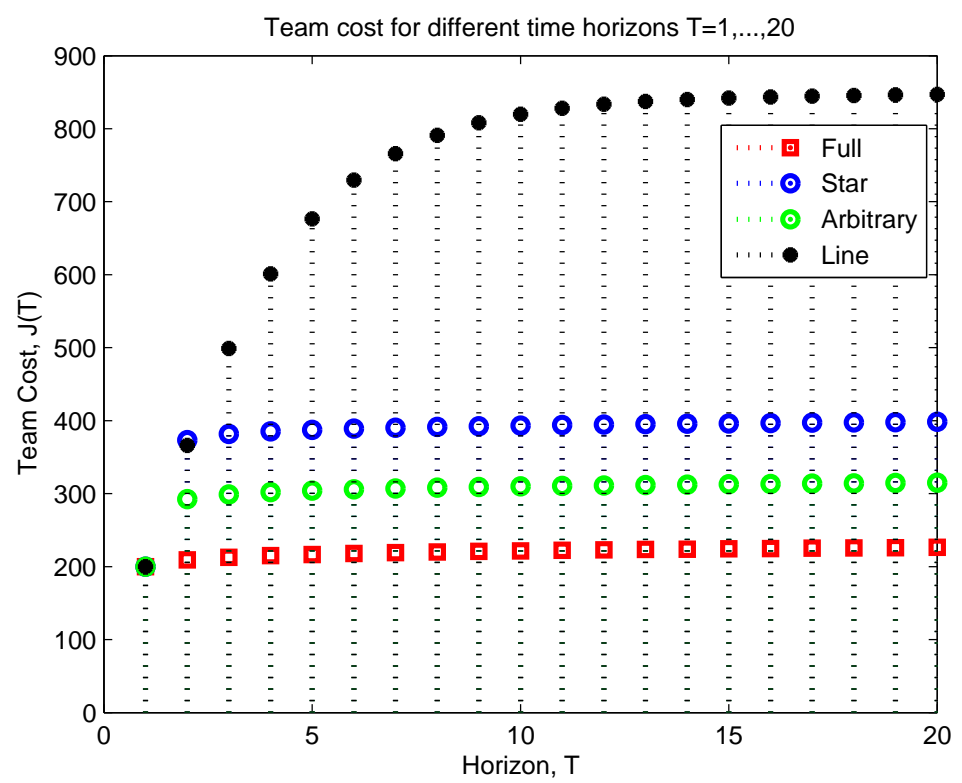

Figure 9: Comparison of oracle cost for different time horizons over certain networks.

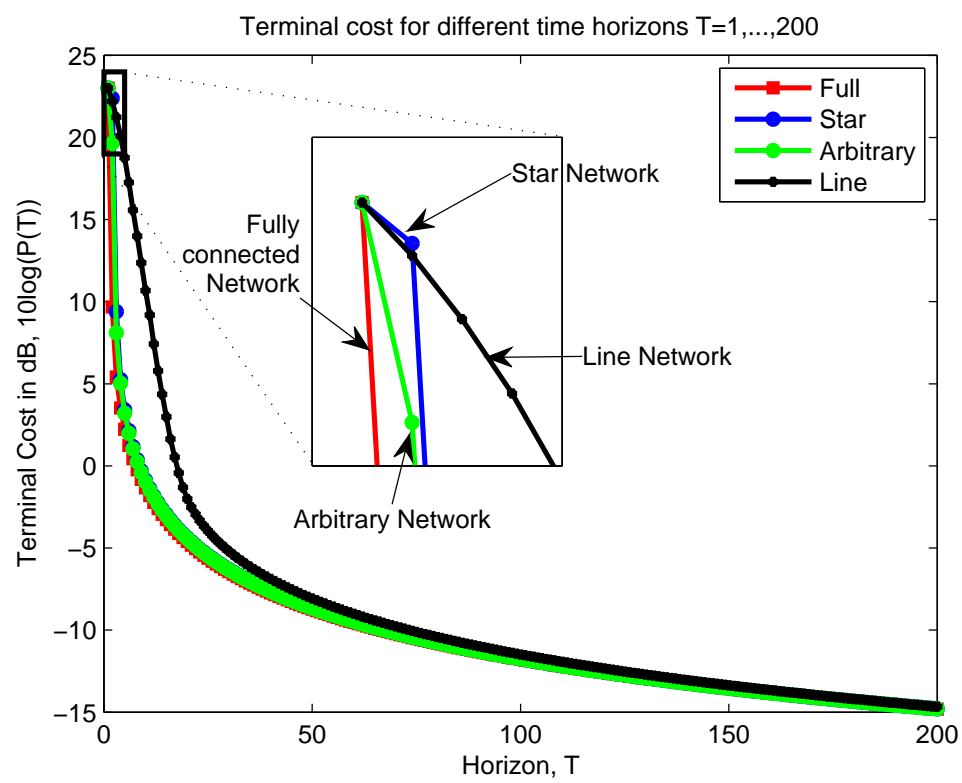

Figure 10: Comparison of terminal cost for different time horizons over certain networks. 
i.e., $J(T)$ is a non-decreasing function of $T$ (indeed increasing function since

Importantly, in Figs. 9 and 10, the oracle and terminal costs lead to counterintuitive results as time horizon grows. In particular, while the oracle costs over different networks are separated as time horizon grows, the terminal costs 


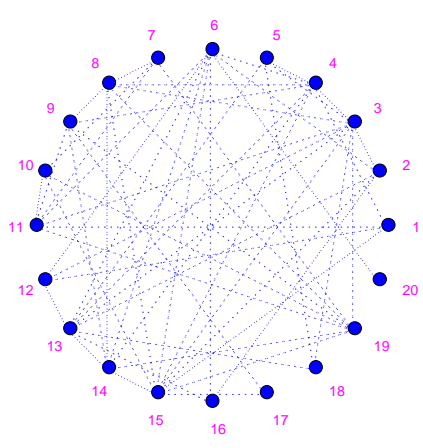

(a) Arbitrary network

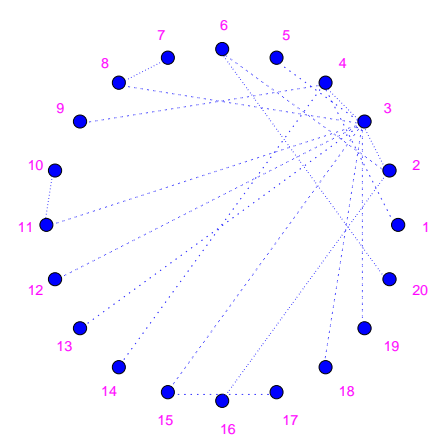

(b) Corresponding spanning tree

Figure 11: An arbitrary network and a corresponding spanning tree.

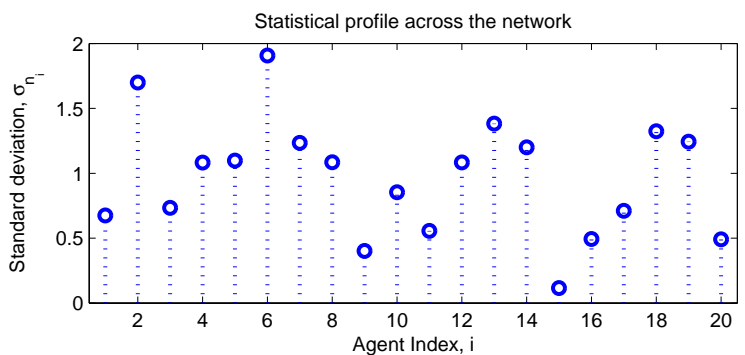

Figure 12: Standard deviation of measurement noise across the network in Fig. 11 significant role in these distinct results. By yielding relatively large cost, the actions in the early stages have crucial impact on the team cost. Therefore, the sequential algorithms aiming to learn the underlying state in time through a trial and error based approach lead to larger team cost over horizon since trials at early stages lead to significantly large costs. In the following, we analyze such circumstances.

We consider a randomly generated network in Fig. 11a constructed as the network in Fig. 7c. This network is not a tree and has loops. We construct a spanning tree of this network, see Fig. $11 \mathrm{~b}$, based on the paths from the agent having the largest neighborhood size, i.e., $i=\arg \max _{j} \pi_{j}$, and then eliminating the multi-paths [21]. The statistical profile of the measurement noise is plotted 


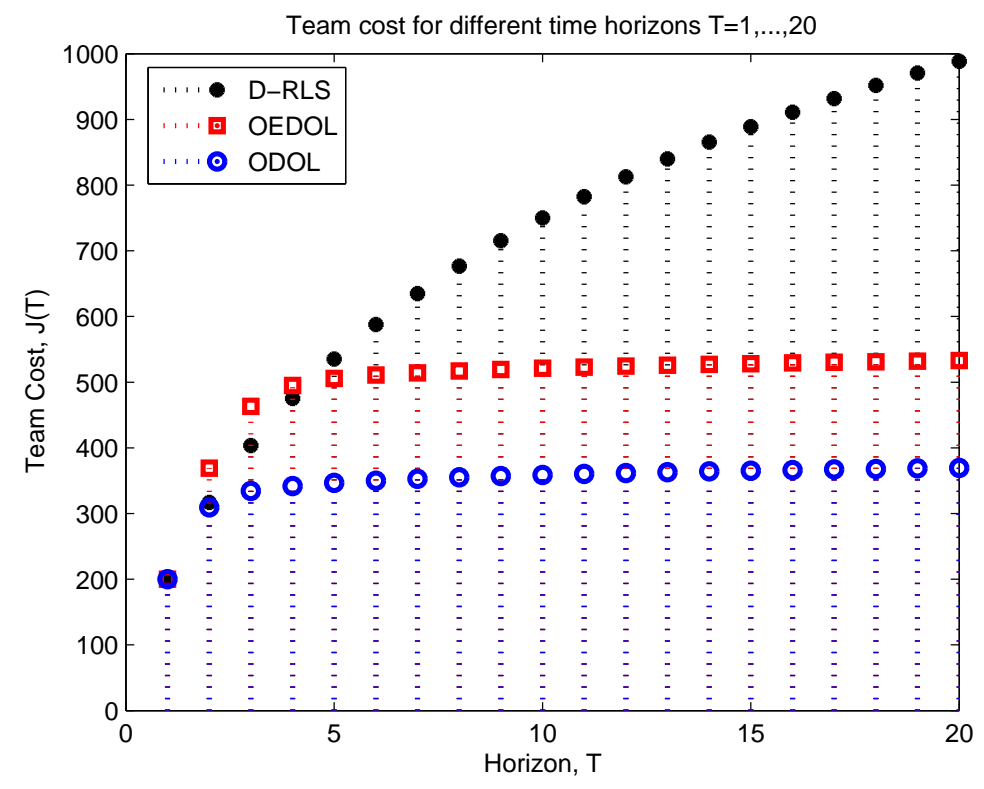

Figure 13: Comparison of team cost of the algorithms for different time horizon.

in Fig. 12 Here, we consider the diffusion recursive least squares (D-RLS) algorithm [29] with Laplacian combination rule [30] for the incremental update and relative-variance combination rule for the spatial update. In D-RLS, agents use a recursive least squares (RLS) update locally, diffuse local estimate to the neighbors at each time instant, and combine received estimates and the local one linearly through certain combination weights, e.g., agent- $i$ has the weight $\lambda_{i, j}$ for the information received from the neighbor- $j$ :

$$
\lambda_{i, j}= \begin{cases}\frac{\sigma_{n_{j}}^{-2}}{\sum_{k \in \mathcal{N}_{i} \cup\{i\}} \sigma_{n_{k}}^{-2}} & \text { if } j \in \mathcal{N}_{i} \cup\{i\} \\ 0 & \text { otherwise. }\end{cases}
$$

In Fig. 13, we compare the ensemble average of the empirical-cost (over 535 100 independent trials) of the ODOL and D-RLS algorithms over Fig. 11a and the OEDOL algorithm over Fig. $11 b$ for different time horizon. Note that in the OEDOL algorithm, agents exchange local estimates while in the ODOL algorithm, agents exchange information through time-stamped approach. The 


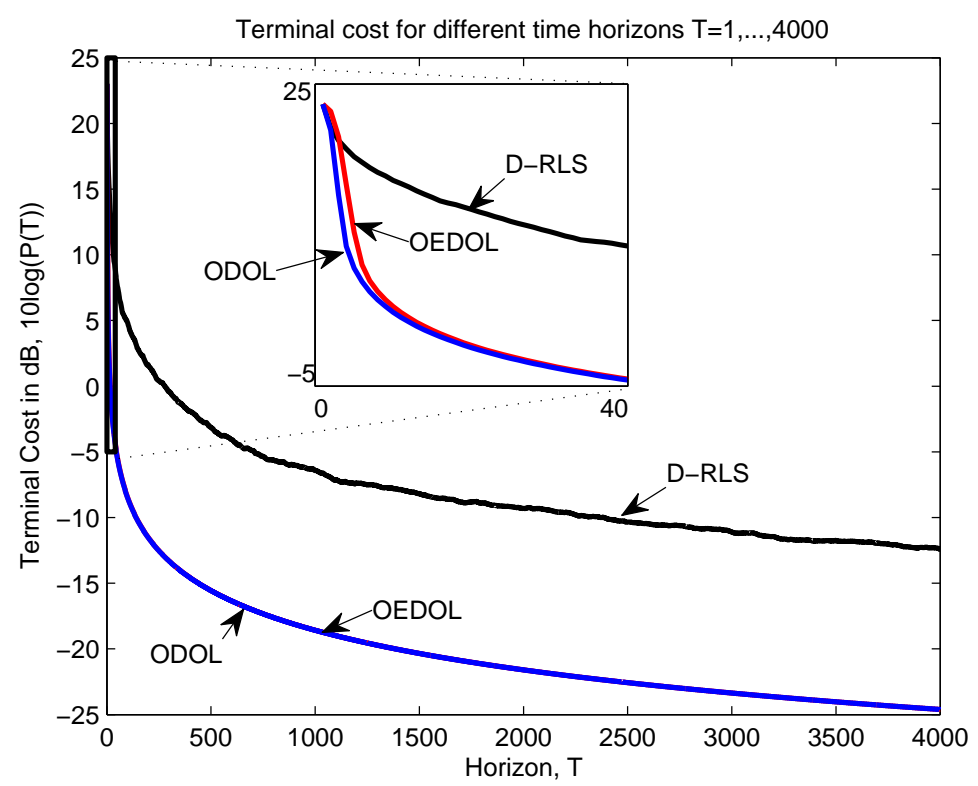

Figure 14: Comparison of terminal cost of the algorithms for different time horizon.

OEDOL algorithm can achieve the oracle performance over a tree network.

Therefore, we construct a spanning tree of the arbitrarily generated network and the OEDOL algorithm achieves the oracle performance of the spanning tree. Since we eliminate certain links while constructing the spanning tree, the oracle performance, i.e., team-cost, is worse than the oracle performance over the original network. However, even though the OEDOL algorithm operates over the spanning tree, the algorithm has performed better than the D-RLS algorithm operating over the original network. Additionally, in Fig. 14, we compare the terminal cost of the algorithms for larger time horizon, e.g., $T=1, \cdots, 4000$. We observe that even though the OEDOL algorithm operates over the spanning tree, the terminal costs of the ODOL and OEDOL algorithms are close to each other for large time horizon, e.g., $T>40$. We also note that as $T \rightarrow \infty$, the terminal cost decreases since noise processes are white.

Finally, in Fig. 15 we examine the impact of the time-windowing depth, e.g., $T_{o}=50,100$, on the terminal cost for different time horizon over an arbitrarily 


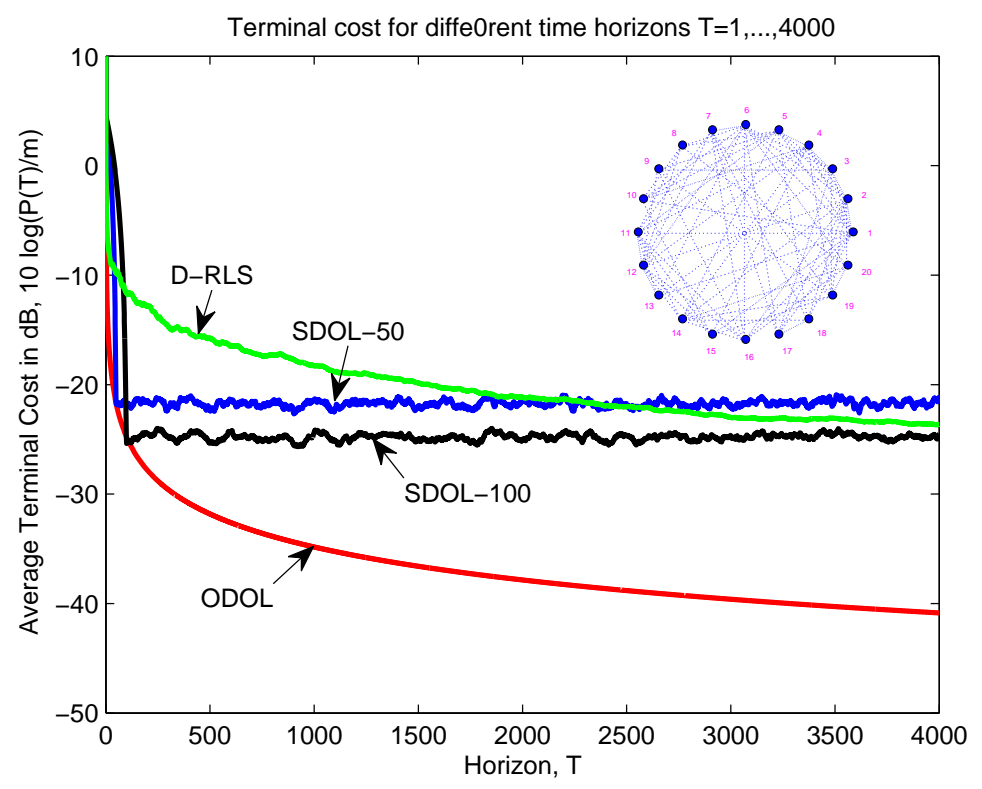

Figure 15: Comparison of terminal cost of the SDOL-50 and SDOL-100 algorithms.

generated network of 20 agents seen on the upper-right of Fig. 15. We note out that for $T<T_{o}$, the terminal cost of the SDOL algorithms differs from the terminal cost of the ODOL algorithm, i.e., the oracle performance since in the SDOL algorithms, agents uses the update 44 for $t<T_{o}$ assuming that all measurements $y_{j, \tau}=0$ for $j=1, \cdots, m$ and $\tau<1$. As also pointed out in Remark 4.1, by excluding the impact of these measurements on the future actions through sliding time-window, the terminal cost of the SDOL- $T_{o}$ algorithm eventually reaches the terminal cost of the ODOL algorithm at $T=$ $T_{o}$ and for larger time window, this performance is maintained. Furthermore, depending on the time window depth $T_{o}$, the agents can achieve smaller terminal cost.

\section{Conclusion}

Distributed algorithms have attracted significant attention due to their wide spread applicability to highly complex structures from biological systems to so- 
cial and economical networks. However, there are still challenges for disclosure and utilization of information among agents. We have considered this problem as a team problem, in which each agent takes actions, e.g., which information to disclose and how to construct the local estimate. We introduced the ODOL algorithm achieving the oracle cost for Gaussian state and noise statistics through a time-stamped approach. Importantly, we have shown that the exchange of the local estimates is sufficient to achieve the oracle cost only over certain network topologies, e.g., over the introduced tree networks involving cell structures. Furthermore, we have introduced the OEDOL algorithm, which achieves the oracle cost through the exchange of local estimates over tree networks. Finally, we have introduced a time-windowing approach for practical applications due to reduced complexity. The sub-optimal approaches possess recursive updates with time-invariant combination weights that should be calculated only once.

Some future directions of research on this topic include team problems for distributed MMSE estimation of a dynamic state and formulation of optimal information exchange in team decision problems for linear continuous-time systems [31].

\section{Acknowledgments}

This work is supported in part by TUBITAK Contract No 115E917, in part by the U.S. Office of Naval Research (ONR) MURI grant N00014-16-1-2710, and in part by NSF under grant CCF 11-11342.

\section{References}

[1] M. K. Banavar, J. J. Zhang, B. Chakraborty, H. Kwon, Y. Li, H. Jiang, A. Spanias, C. Tepedelenlioglu, C. Chakrabarti, A. P.-Suppappola, An overview of recent advances on distributed and agile sensing algorithms and implementation, Digital Signal Processing 39 (2015) 1-14.

[2] A. H. Sayed, S.-Y. Tu, J. Chen, X. Zhao, Z. J. Towfic, Diffusion strategies for adaptation and learning over networks: An examination of distributed 
strategies and network behavior, IEEE Signal Processing Magazine 30 (3) (2013) 155-171.

[3] S. Kar, J. M. F. Moura, Consensus+innovations distributed inference over networks: Cooperation and sensing in networked systems, IEEE Signal Processing Magazine 30 (3) (2013) 99-109.

[4] J. Fernandez-Bes, L. A. Azpicueta-Ruiz, J. Arenas-Garcia, M. T. M. Silva, Distributed estimation in diffusion networks using affine least-squares combiners, Digital Signal Processing 36 (2015) 1-14.

[5] V. Krishnamurthy, Decentralized activation in sensor networks-global games and adaptive filtering games, Digital Signal Processing 21 (5) (2011) 638-647.

[6] A. Jadbabaie, P. Molavi, A. Sandroni, A. Tahbaz-Salehi, Non-Bayesian social learning, Games and Economic Behavior 76 (1) (2012) 210-225.

[7] D. Acemoglu, A. Ozdaglar, Dynamic games and applications, Opinion dynamics and learning in social networks 1 (1) (2011) 3-49.

[8] M. Jackson, Social and Economic Networks, Princeton University Press, Princeton, N.J., 2008.

[9] C. G. Lopes, A. H. Sayed, Diffusion least-mean squares over adaptive networks: Formulation and performance analysis, IEEE Transactions on Signal Processing 56 (7) (2008) 3122-3136.

[10] F. S. Cattivelli, A. H. Sayed, Diffusion strategies for distributed Kalman filtering and smoothing, IEEE Transactions on Automatic Control 55 (9) (2010) 2069-2084.

[11] S. Shahrampour, A. Rakhlin, A. Jadbabaie, Online learning of dynamic parameters in social networks, in: Neural Information Processing Systems, 2013, pp. 2013-2021. 
[12] G. Mateos, I. D. Schizas, G. B. Giannakis, Distributed recursive leastsquares for consensus-based in-network adaptive estimation, IEEE Transactions on Signal Processing 57 (11) (2009) 4583-4588.

[13] M. O. Sayin, S. S. Kozat, Single bit and reduced dimension diffusion strategies over distributed networks, IEEE Signal Processing Letters 20 (10) (2013) 976-979.

[14] M. O. Sayin, S. S. Kozat, Compressive diffusion strategies over distributed networks for reduced communication load, IEEE Transactions on Signal Processing 62 (20) (2014) 5308-5323.

[15] V. D. Blondel, J. M. Hendrickx, A. Olshevsky, J. N. Tsitsiklis, Convergence in multiagent coordination, consensus, and flocking, in: Proceedings of 44th IEEE Conference on Decision and Control (CDC-ECC), 2005, pp. 29963000 .

[16] D. S. Scherber, H. C. Papadopoulos, Locally constructed algorithms for distributed computations in ad-hoc networks, in: Proceedings of Information Processing Sensor Networks (IPSN), 2004, pp. 11-19.

[17] N. Metropolis, A. W. Rosenbluth, M. N. Rosenbluth, A. H. Teller, E. Teller, Equation of state calculations by fast computing machines, The Journal of Chemical Physics 21 (6) (1953) 1087-1092.

[18] A. H. Sayed, Fundamentals of Adaptive Filtering, Wiley, New York, 2003.

[19] B. D. O. Anderson, J. B. Moore, Optimal Filtering, Prentice-Hall, Inc., Englewood Cliffs, NJ, 1979.

[20] S. Skiena, Implementing Discrete Mathematics: Combinatorics and Graph Theory with Mathematica, Addison-Wesley, Reading, MA, 1990.

[21] B. Y. Wu, K.-M. Chao, Spanning Trees and Optimization Problems (Discrete Mathematics and Its Applications), CRC Press, New York, 2004. 
[22] R. G. Gallager, P. A. Humblet, P. M. Spira, A distributed algorithm for minimum-weight spanning trees, ACM Transactions on Programming Languages and Systems 5 (1) (1983) 66-71.

[23] D. Peleg, Distributed Computing: A Locality-Sensitive Approach, SIAM, Philadelphia, PA, 2000.

[24] M. Elkin, Unconditional lower bounds on the time-approximation tradeoffs for the distributed minimum spanning tree problem, in: Proceedings of 36th ACM Symposium on Theory of Computing (STOC), 2004, pp. 331-340.

[25] M. Khan, G. Pandurangan, V. S. A. Kumar, Distributed algorithms for constructing approximate minimum spanning trees in wireless sensor networks, IEEE Transactions on Parallel and Distributed Systems 20 (1) (2009) 124139.

[26] J. Chen, A. H. Sayed, Optimal combination rules for adaptation and learning over networks, in: Proceedings of 50th Annual Allerton Conference on Communication, Control, and Computing (Allerton), 2012, pp. 1535-1542.

[27] J. Chen, A. H. Sayed, On the learning behavior of adaptive networks - Part II: transient analysis, IEEE Transactions on Information Theory 61 (6).

[28] J. Chen, A. H. Sayed, On the learning behavior of adaptive networks - Part II: performance analysis, IEEE Transactions on Information Theory 61 (6).

[29] F. S. Cattivelli, C. G. Lopes, A. H. Sayed, Diffusion recursive least-squares for distributed estimation over adaptive networks, IEEE Transactions on Signal Processing 56 (5) (2008) 1865-1877.

[30] R. Olfati-Saber, R. M. Murray, Consensus problems in networks of agents with switching topology and time-delays, IEEE Transactions on Automatic Control 49 (9) (2004) 1520-1533.

[31] A. Bagchi, T. Başar, Team decision theory for linear continuous-time systems, IEEE Transactions on Automatic Control AC-25 (6) (1980) 11541161. 